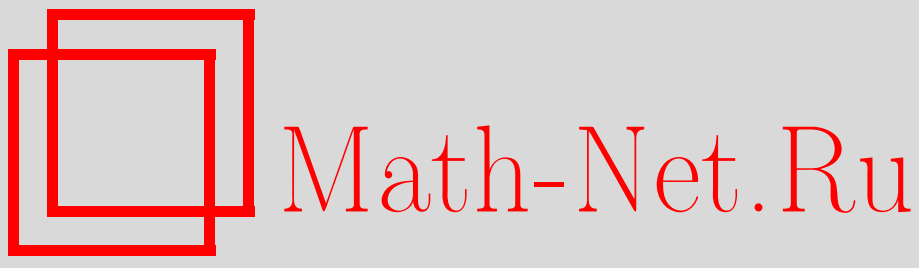

А. Я. Мальцев, Наследование гамильтоновых структур в методе усреднения Уизема, Изв. РАН. Сер. матем., 1999, том 63, выпуск 6, 117-146

DOI: https://doi.org/10.4213/im269

Использование Общероссийского математического портала Math-Net.Ru подразумевает, что вы прочитали и согласны с пользовательским соглашением http://www. mathnet.ru/rus/agreement

Параметры загрузки:

IP : 52.90 .164 .192

26 апреля 2023 г., $17: 16: 41$ 
УДК 514.944

\author{
А.Я. Мальцев
}

\title{
Наследование гамильтоновых структур в методе усреднения Уизема
}

\begin{abstract}
В работе рассматривается метод усреднения Уизема для систем, обладающих локальной теоретико-полевой гамильтоновой структурой. Дается доказательство сохранения таких гамильтоновых структур для усредненной системы. Рассмотрение базируется на предложенной ранее Б. А. Дубровиным и С. П. Новиковым процедуре усреднения скобки Пуассона, для которой устанавливаются необходимые свойства сохранения тождества Якоби и инвариантности.

Библиография: 16 наименований.
\end{abstract}

\section{Введение}

Данная работа посвящена методу Уизема, позволяющему исследовать эволюцию медленно промодулированных $m$-фазных решений нелинейных систем уравнений (см. [1], а также [2], [4]-[6], [8]). Параметры $m$-фазных решений (например, "бегуших волн" при $m=1$ ) удовлетворяют при этом квазилинейным однородным эволюционным системам первого порядка, т.е. системам вида

$$
U_{T}^{i}=V_{j}^{i}(\mathbf{U}) U_{X}^{j}, \quad i, j=1, \ldots, N, \quad \mathbf{U}=\left(U^{1}, \ldots, U^{N}\right) .
$$

При изучении таких систем оказывается важным исследование их гамильтоновости относительно локальной скобки Пуассона гидродинамического типа [3], [4]. Теория таких скобок, построенная Б. А. Дубровиным и С. П. Новиковым, была успешно использована С. П. Царевым [9] для интегрирования систем (1), имеюших гамильтонову структуру при условии существования координат $\overline{\mathbf{U}}=\overline{\mathbf{U}}(\mathbf{U})$, в которых матрища $V_{j}^{i}$ диагональна. Исследование гамильтоновых систем, не удовлетворяющих этому условию, было проведено Е. В. Ферапонтовым [10], [11]. В работе [4] была предложена также процедура построения гамильтоновой структуры требуемого вида для системы уравнений Уизема (1) при условии гамильтоновости исходной системы относительно локальной теоретико-полевой скобки Пуассона, однако доказательство тождества Якоби для получаемой при этом скобки отсутствовало, о чем сообшалось в [12]. В данной работе мы докажем справедливость тождества Якоби для получаемой таким образом скобки в случае общего положения, пользуясь процедурой ограничения по Дираку скобки Пуассона на подмногообразие в функциональном пространстве, что и позволит сделать утверждение о наследовании (локальных) гамильтоновых структур в методе Уизема. Отметим также, что для систем, допускаюших локальньй лагранжев формализм, связанный со скобкой Пуассона, справедливость процедуры Дубровина-Новикова была установлена в [16].

Работа выполнена при финансовой поддержке KFA Forschungzentrum Jülich GmbH (грант INTAS № 96-0770) и Российского фонда фундаментальных исследований (грант № 96-01-01623).

(C) А.Я. МАльцев 1999 


\section{§ 1. Общие конструкции}

Пусть имеется эволюционная система на пространстве полей $\varphi=\left(\varphi^{1}, \ldots, \varphi^{n}\right)$ вида

$$
\varphi_{t}^{i}=Q^{i}\left(\boldsymbol{\varphi}, \varphi_{x}, \varphi_{x x}, \ldots\right), \quad i=1, \ldots, n,
$$

являющаяся гамильтоновой по отношению к локальной теоретико-полевой скобке Пуассона:

$$
\left\{\varphi^{i}(x), \varphi^{j}(y)\right\}=\sum_{k \geqslant 0} B_{k}^{i j}\left(\varphi, \varphi_{x}, \ldots\right) \delta^{(k)}(x-y)
$$

(в сумме присутствует конечное число слагаемых), с гамильтонианом

$$
H=\int \mathscr{P}_{H}\left(\boldsymbol{\varphi}, \boldsymbol{\varphi}_{x}, \ldots\right) d x
$$

Скобка (3) может быть совместима с оператором трансляции, т.е. может существовать локальньй полевой функционал

$$
P=\int \mathscr{P}_{P}\left(\boldsymbol{\varphi}, \boldsymbol{\varphi}_{x}, \ldots\right) d x
$$

называемый оператором импульса, такой, что $\left\{\varphi^{i}(x), P\right\}=\varphi_{x}^{i}$. (Как указал автору О. И. Мохов, этим свойством обладает специальный класс скобок указанного вида; см. [13], [14]. Однако в физических гамильтоновых системах это свойство, как правило, присутствует.) Кроме того, мы будем допускать, что скобка (3) может иметь конечное число аннуляторов, т.е. таких функционалов (не обязательно имеющих вид (4), (5)) $N_{1}, \ldots, N_{p}$, что в силу (3) $\left\{\varphi^{i}(x), N_{q}\right\}=0$.

ОПРЕДЕЛЕНИЕ. Пусть имеются функция $m$ переменных $\boldsymbol{\Phi}(\theta)=\left(\Phi^{i}\left(\theta_{1}, \ldots, \theta_{m}\right)\right)$, $i=1, \ldots, n, 2 \pi$-периодическая по каждому из аргументов, а также соответствующие ей $m$-мерные векторы $\boldsymbol{\omega}=\left(\omega^{1}, \ldots, \omega^{m}\right), \mathbf{k}=\left(k^{1}, \ldots, k^{m}\right)$ такие, что

$$
\omega^{\alpha} \Phi_{\theta^{\alpha}}^{i}=Q^{i}\left(\boldsymbol{\Phi}, k^{\alpha} \boldsymbol{\Phi}_{\theta^{\alpha}}, \ldots\right)
$$

тогда соответствующая ей функция

$$
\varphi(x, t)=\mathbf{\Phi}(\mathbf{k} x+\omega t)
$$

называется $m$-фазным квазипериодическим решением системы (2).

Заметим, что существование $m$-фразных решений с $m>1$ характерно, как правило, лишш для "интегрируемых" систем типа КдФ.

Система (6) представляет собой систему дифференциальных уравнений в частных производных на функции $\boldsymbol{\Phi}(\theta) \quad\left(\theta=\left(\theta^{1}, \ldots, \theta^{m}\right)\right)$, ее $2 \pi$-периодические по всем переменным решения (если они есть) при всевозможных $\mathbf{k}$ и $\boldsymbol{\omega}$ дают полное семейство $m$-ффазных решений системы (2).

Мы будем теперь предполагать, что при любых $\mathbf{k}$ и $\boldsymbol{\omega}$ (подразумевается, что система (2) не является линейной) система (2) имеет некоторое семейство требуемых решений, параметризуемое сдвигами начальных фаз $\theta_{0}^{\alpha}$, а также, возможно, некоторыми дополнительными параметрами $r^{1}, \ldots, r^{g}$, неизменными при сдвигах начальных фаз, число которых постоянно при всех $\boldsymbol{\omega}$ и $\mathbf{k}$. Таким образом, $m$-фазные решения (2) будут задаваться значениями величин $\boldsymbol{\omega}=\left(\omega^{1}, \ldots, \omega^{m}\right)$, $\mathbf{k}=\left(k^{1}, \ldots, k^{m}\right), r^{1}, \ldots, r^{g}$, а также $\theta_{0}^{1}, \ldots, \theta_{0}^{m}$. 
Пусть теперь система (2) имеет $N=2 m+g$ транслящионно инвариантных (т.е. коммутируюших с оператором сдвига по $x$ ) первых интегралов вида

$$
I^{\nu}=\int \mathscr{P}^{\nu}\left(\varphi, \varphi_{x}, \ldots\right) d x, \quad \nu=1, \ldots, N
$$

коммутируюших с гамильтонианом и находяшихся в инволюции относительно скобки (3), т.е.

$$
\left\{I^{\nu}, I^{\mu}\right\}=0 .
$$

Среди интегралов (8) могут присутствовать гамильтониан, импульс, а также аннуляторы скобки.

Мы будем предполагать, что параметры $\mathbf{k}, \boldsymbol{\omega}$ и $\mathbf{r}=\left(r^{1}, \ldots, r^{g}\right)$ функционально независимы на семействе $m$-фазных решений (2) и, кроме того, могут быть однозначно выражены через значения интегралов (8) на соответствуюших им функциях семейства (7), т.е. $\mathbf{k}=\mathbf{k}(\mathbf{U}), \boldsymbol{\omega}=\boldsymbol{\omega}(\mathbf{U}), \mathbf{r}=\mathbf{r}(\mathbf{U}), \mathbf{U}=\left(U^{1}, \ldots, U^{N}\right)$, где

$$
\begin{aligned}
U^{\nu} & =\lim _{T \rightarrow \infty} \frac{1}{2 T} \int_{-T}^{T} \mathscr{P}^{\nu}\left(\boldsymbol{\varphi}, \boldsymbol{\varphi}_{x}, \ldots\right) d x \\
& =\frac{1}{(2 \pi)^{m}} \int_{0}^{2 \pi} \cdots \int_{0}^{2 \pi} \mathscr{P}^{\nu}\left(\boldsymbol{\Phi}, k^{\alpha} \boldsymbol{\Phi}_{\theta^{\alpha}}, \ldots\right) d^{m} \theta
\end{aligned}
$$

(легко видеть, что интегралы (10) не зависят от начальных фаз $\theta_{0}^{\alpha}$ ). Таким образом, выбрав при каждом значении параметров $\mathbf{U}$ на $\mathscr{M}$ какую-то определенную функцию $\boldsymbol{\Phi}_{\text {in }}(\theta, \mathbf{U})$, имеюшую нулевые начальные фазы $\theta_{0}$ (гладким образом), параметрами на семействе $m$-фазных решений (2) можно считать величины $U^{\nu}$ и $\theta_{0}^{\alpha}$.

Каждый из функционалов (8) порождает в силу скобки (3) гамильтонов поток вида

$$
\varphi_{\tau^{\nu}}^{i}=Q_{\nu}^{i}\left(\boldsymbol{\varphi}, \varphi_{x}, \ldots\right) .
$$

Все эти потоки коммутируют между собой и с потоком (2) и оставляют семейства $m$-фазных решений (2) инвариантными, порождая на них линейную зависимость начальных фаз $\theta_{0}^{\alpha}$ от времен $\tau^{\nu}$ и оставляя неизменными остальные параметры; т.е. сушествуют функции $\omega_{\nu}^{\alpha}(\mathbf{U})$ такие, что для любой функции $\boldsymbol{\Phi}\left(\theta+\theta_{0}, \mathbf{U}\right)$ семейства $m$-фазных решений (2) имеет место соотношение

$$
\omega_{\nu}^{\alpha}(\mathbf{U}) \Phi_{\theta^{\alpha}}^{i}=Q_{\nu}^{i}\left(\boldsymbol{\Phi}, k^{\alpha} \boldsymbol{\Phi}_{\theta^{\alpha}}, \ldots\right) .
$$

В силу (9) эволюционные системы (11), порожденные интегралами $I^{\nu}$, обладают теми же свойствами, что и система (2), и таким образом все утверждения, доказанные для системы (2), верны и для систем (11), не являюшихся нулевыми потоками и оператором сдвига по $x$, о чем будет сказано при рассмотрении метода Уизема, в котором существенна нелинейность системы.

Система (6) при каждых $\boldsymbol{\omega}$ и $\mathbf{k}$ определяет некоторое "подмногообразие" (обозначим его $\mathscr{M}_{\boldsymbol{\omega}, \mathbf{k}}$ ) в пространстве $2 \pi$-периодических по всем $\theta^{\alpha}$ функций. При этом функционалы

$$
F_{\boldsymbol{\omega}, \mathbf{k}}^{i}[\mathbf{\Phi}](\theta)=\omega^{\alpha} \Phi_{\theta^{\alpha}}^{i}-Q^{i}\left(\mathbf{\Phi}, k^{\alpha} \mathbf{\Phi}_{\theta^{\alpha}}, \ldots\right)
$$

являются "связями", определяюшими это подмногообразие.

Мы будем предполагать, что в случае, когда оператор $I^{\nu}$ не является оператором импульса или аннулятором скобки (3), соответствуюшая ему система (12) при 
всевозможных $\omega_{\nu}$ и $\mathbf{k}$, так же, как и $(6)$, определяет полное семейство $m$-фазных решений (2), т.е. система связей

$$
F_{\boldsymbol{\omega}_{\nu}, \mathbf{k}}^{i(\nu)}[\boldsymbol{\Phi}](\theta)=\omega_{\nu}^{\alpha} \Phi_{\theta^{\alpha}}^{i}-Q_{\nu}^{i}\left(\boldsymbol{\Phi}, k^{\alpha} \boldsymbol{\Phi}_{\theta^{\alpha}}, \ldots\right)=0
$$

определяет при каждых $\boldsymbol{\omega}_{\nu}$ и $\mathbf{k}$ некоторое $(m+g)$-параметрическое семейство $\mathscr{M}_{\boldsymbol{\omega}_{\nu}, \mathbf{k}}^{\nu} 2 \pi$-периодических по всем $\theta^{\alpha}$ решений, параметризуемое сдвигами начальных фаз и некоторыми дополнительными параметрами, число которых равно $g$, так что объединение всех $\mathscr{M}_{\boldsymbol{\omega}_{\nu}, \mathbf{k}}^{\nu}$ при всевозможных $\boldsymbol{\omega}_{\nu}$ и $\mathbf{k}$ дает полное семейство $m$-фазных решений (2) (это выполняется в известных примерах).

Далее в описываемой ситуации мы будем считать (это имеет место в приме$\operatorname{pax})$, что количество $g$ параметров $r^{1}, \ldots, r^{g}$ равно числу $p$ аннуляторов скобки (3), для чего имеется следующая мотивация: любые $m+1$ потоков $(11)$, порождаемые произвольными $m+1$ интегралами (8), линейно зависимы в силу (12) на семействе $m$-фазных решений $(2)$, т.е. сушествуют $\lambda^{1}(\mathbf{U}), \ldots, \lambda^{m+1}(\mathbf{U})$ такие, что $\sum_{j=1}^{m+1}\left(\lambda^{j}\right)^{2}=1$ и $\sum_{j=1}^{m+1} \lambda^{j}(\mathbf{U}) \omega_{\nu_{j}}^{\alpha}(\mathbf{U})=0 \forall \alpha$. Отсюда следует, что $m$-фразные решения системы (2) можно определить также соотношением

$$
\delta \sum_{j=1}^{m+1} \lambda^{j} I^{\nu_{j}}=\delta \sum_{q=1}^{p} \mu^{q} N_{q}
$$

(где $N^{q}$ - аннуляторы скобки (3), поскольку функционал $\sum_{j=1}^{m+1} \lambda^{j}(\mathbf{U}) I^{\nu_{j}}$ порождает на соответствуюших $m$-фазных решениях нулевой поток) на функциях вида

$$
\varphi(x)=\boldsymbol{\Phi}(\mathbf{k} x),
$$

где $\boldsymbol{\Phi}(\theta)=\boldsymbol{\Phi}\left(\theta^{1}, \ldots, \theta^{m}\right)-2 \pi$-периодическая функция $\theta$. Предполагая, что при любых $\mathbf{k}=\left(k^{1}, \ldots, k^{m}\right), \mu^{1}, \ldots, \mu^{p}$ и $\lambda^{1}, \ldots, \lambda^{m+1}$, связанных соотношением $\sum_{j=1}^{m+1}\left(\lambda^{j}\right)^{2}=1$, функционал $\sum_{j=1}^{m+1} \lambda^{j} I^{\nu_{j}}-\sum_{q=1}^{p} \mu^{q} N_{q}$ имеет на функциях (15) (с точностью до сдвигов начальных фаз $\theta_{0}^{\alpha}$ ) единственный экстремум, получим, что $m$-фазные решения (2) характеризуются $2 m+p$ независимыми параметрами, не считая начальных фаз; отсюда и следует сформулированное выше предположение.

По аналогии с конечномерным случаем мы потребуем от многообразий $\mathscr{M}_{\boldsymbol{\omega}, \mathbf{k}}$ и $\mathscr{M}_{\boldsymbol{\omega}_{\nu}, \mathbf{k}}^{(\nu)}$ (для $I^{\nu}$, не являющихся аннуляторами и оператором импульса) выполнения некоторого свойства регулярности, которое в конечномерном случае выражается полнотой ранга матрищы производных от связей. А именно, линеаризуем функционал (13) $\mathbf{F}_{\boldsymbol{\omega}, \mathbf{k}}(\theta)=\left(F_{\boldsymbol{\omega}, \mathbf{k}}^{1}(\theta), \ldots, F_{\boldsymbol{\omega}, \mathbf{k}}^{n}(\theta)\right)$ (соответственно любой из функционалов $(14))$ на решении $\boldsymbol{\Phi}_{\text {in }}\left(\theta+\theta_{0}, U\right)$ системы (12) (оно будет также и решением (14)), т.е. введем оператор $\hat{\mathbf{L}}_{\mathbf{U}, \theta_{0}}\left(\right.$ соответственно $\hat{\mathbf{L}}_{\mathbf{U}, \theta_{0}}^{(\nu)}$ ) с ядром $L_{\mathbf{U}}^{i j}\left(\theta+\theta_{0}, \theta^{\prime}+\theta_{0}\right)\left(L_{\mathbf{U}}^{i j(\nu)}\left(\theta+\theta_{0}, \theta^{\prime}+\theta_{0}\right)\right)$ такой, что

$$
\begin{aligned}
& \delta F_{\boldsymbol{\omega}(\mathbf{U}), \mathbf{k}(\mathbf{U})}^{i}(\theta)= \\
& \quad=\frac{1}{(2 \pi)^{m}} \int_{0}^{2 \pi} \cdots \int_{0}^{2 \pi} \sum_{j} L_{\mathbf{U}}^{i j}\left(\theta+\theta_{0}, \theta^{\prime}+\theta_{0}\right)\left(\Phi^{j}\left(\theta^{\prime}\right)-\Phi_{\text {in }}^{j}\left(\theta^{\prime}+\theta_{0}, \mathbf{U}\right)\right) d^{m} \theta \\
& \quad=\left(\hat{\mathbf{L}}_{\mathbf{U}, \theta_{0}} \delta \boldsymbol{\Phi}\right)^{i}(\theta)
\end{aligned}
$$

(аналогично для связей $\left.F_{\boldsymbol{\omega}_{\nu}, \mathbf{k}}^{i(\nu)}[\boldsymbol{\Phi}](\theta)\right)$. 
Так определенные $\hat{\mathbf{L}}_{\mathbf{U}, \theta_{0}}\left(\hat{\mathbf{L}}_{\mathbf{U}, \theta_{0}}^{(\nu)}\right)$ являются дифференциальными по $\theta$ операторами с периодическими коэффициентами в пространстве $2 \pi$-периодических функций $\Phi^{i}(\theta), i=1, \ldots, n$. Мы потребуем выполнения следуюших условий.

А) При любых $\mathbf{U}$ и $\theta_{0}$ ядро $\mathbf{L}_{\mathbf{U}, \theta_{0}}$ оператора (16) состоит из векторов, касательных к задаваемому системой (12) подмногообразию $\mathscr{M}_{\boldsymbol{\omega}(\mathbf{U}), \mathbf{k}(\mathbf{U})}$, т.е. функции $\boldsymbol{\Phi}_{\theta^{\alpha}}(\theta, \mathbf{k}, \boldsymbol{\omega}, \mathbf{r})$ и $\boldsymbol{\Phi}_{r \zeta}(\theta, \mathbf{k}, \boldsymbol{\omega}, \mathbf{r})$ дают базис в пространстве всех решений системы

$$
\left(\hat{\mathbf{L}}_{\mathbf{U}, \theta_{0}} \delta \boldsymbol{\Phi}\right)(\theta)=0
$$

и аналогичные свойства имеют место для операторов $\hat{\mathbf{L}}_{\mathbf{U}, \theta_{0}}^{(\nu)}$ и подмногообразий $\mathscr{M}_{\boldsymbol{\omega}_{\nu}(\mathbf{U}), \mathbf{k}(\mathbf{U})}$, соответствуюших $I^{\nu}$ и не являюшихся аннуляторами скобки (3) и оператором импульса.

В) Коразмерность образа операторов $\hat{\mathbf{L}}_{\mathbf{U}, \theta_{0}}, \hat{\mathbf{L}}_{\mathbf{U}, \theta_{0}}^{(\nu)}$ в пространстве $2 \pi$-периодических по $\theta$ функций равна размерности их ядер, значит, все $\hat{\mathbf{L}}_{\mathbf{U}, \theta_{0}}, \hat{\mathbf{L}}_{\mathbf{U}, \theta_{0}}^{(\nu)}$ имеют ровно $m+g(g=p)$ левых собственных векторов (обозначим их $\boldsymbol{\kappa}_{\mathbf{U}, \theta_{0}}^{(s)}$ и $\left.\boldsymbol{\kappa}_{\mathbf{U}, \theta_{0}}^{(s),(\nu)}\right)$, т.е. $2 \pi$-периодических по всем $\theta^{\alpha}$ функций $\kappa_{i \mathbf{U}}^{(s)}\left(\theta+\theta_{0}\right), j=1, \ldots, n, s=1, \ldots$ $\ldots, m+g$ и $\kappa_{i \mathbf{U}}^{(s),(\nu)}\left(\theta+\theta_{0}\right)$ таких, что

$$
\begin{array}{r}
\frac{1}{(2 \pi)^{m}} \int_{0}^{2 \pi} \cdots \int_{0}^{2 \pi} \kappa_{i \mathbf{U}}^{(s)}(\theta) L_{\mathbf{U}}^{i j}\left(\theta, \theta^{\prime}\right) d^{m} \theta \equiv 0, \\
\frac{1}{(2 \pi)^{m}} \int_{0}^{2 \pi} \cdots \int_{0}^{2 \pi} \kappa_{i \mathbf{U}}^{(s),(\nu)}(\theta) L_{\mathbf{U}}^{i j(\nu)}\left(\theta, \theta^{\prime}\right) d^{m} \theta \equiv 0 .
\end{array}
$$

При выполнении этих условий мы будем говорить, что подмногообразия $\mathscr{M}_{\boldsymbol{\omega}, \mathbf{k}}$ и $\mathscr{M}_{\boldsymbol{\omega}_{\nu}, \mathbf{k}}^{\nu}$ в пространстве $2 \pi$-периодических функций $\theta$ обладают свойством регулярности. При этом, предполагая также, что векторы $\boldsymbol{\Phi}_{\theta^{\alpha}}(\theta, \mathbf{k}, \boldsymbol{\omega}, \mathbf{r})$, $\boldsymbol{\Phi}_{r \zeta}(\theta, \mathbf{k}, \boldsymbol{\omega}, \mathbf{r}), \boldsymbol{\Phi}_{k^{\alpha}}(\theta, \mathbf{k}, \boldsymbol{\omega}, \mathbf{r})$ и $\boldsymbol{\Phi}_{\omega^{\beta}}(\theta, \mathbf{k}, \boldsymbol{\omega}, \mathbf{r})$ при любом значении параметров $\left(\theta_{0}, \mathbf{k}, \boldsymbol{\omega}, \mathbf{r}\right)$ линейно независимы (существенное для дальнейших рассуждений требование), объединение вышеописанных подмногообразий $\mathscr{M}_{\boldsymbol{\omega}, \mathbf{k}}$ или $\mathscr{M}_{\boldsymbol{\omega}_{\nu}, \mathbf{k}}^{\nu}$ $(3 m+g=N+m)$ дает $(N+m)$-мерное подмногообразие $\mathscr{M}$ в пространстве $2 \pi$-периодических по всем $\theta^{\alpha}$ функций, соответствуюшее полному набору $m$-фазных решений (2).

\section{§ 2. Метод Уизема}

Описанные выше конструкции тесно связаны с методом усреднения Уизема (см. [1]-[8]) для нелинейных систем уравнений в частных производных (к потокам, порожденным аннуляторами или оператором импульса, он формально не может быть применен), состоящим в следующем: введя малый параметр $\varepsilon$ и положив $T=\varepsilon t$, $X=\varepsilon x$, перепишем систему $(2)$ для полей $\varphi^{i}(X, T)$ в виде

$$
\varepsilon \varphi_{T}^{i}=Q^{i}\left(\boldsymbol{\varphi}, \varepsilon \boldsymbol{\varphi}_{X}, \varepsilon^{2} \boldsymbol{\varphi}_{X X}, \ldots\right)
$$

Рассмотрим теперь систему (19) на пространстве функций $\varphi(\theta, X, T)$, $\theta=\left(\theta^{1}, \ldots, \theta^{m}\right), 2 \pi$-периодических по каждой из переменных $\theta^{\alpha}$. Метод Уизема состоит в нахождении функций $\mathbf{S}(X, T)=\left(S^{1}(X, T), \ldots, S^{m}(X, T)\right)$, 
а также $2 \pi$-периодических по всем $\theta^{\alpha}$ функций $\mathbf{\Phi}(\theta, X, T, \varepsilon)=\left(\Phi^{i}(\theta, X, T, \varepsilon)\right)$, представляемых при $\varepsilon \rightarrow 0$ асимптотическими по $\varepsilon$ рядами

$$
\Phi^{i}(\theta, X, T, \varepsilon)=\sum_{n=0}^{\infty} \varepsilon^{n} \Phi_{(n)}^{i}(\theta, X, T),
$$

таких, что функция

$$
\varphi(\theta, X, T, \varepsilon)=\mathbf{\Phi}\left(\theta+\frac{\mathbf{S}(X, T)}{\varepsilon}, X, T, \varepsilon\right)=\sum_{n=0}^{\infty} \varepsilon^{n} \mathbf{\Phi}_{(n)}\left(\theta+\frac{\mathbf{S}(X, T)}{\varepsilon}, X, T\right)
$$

удовлетворяет системе (19) при любых $\theta$ и $\varepsilon(\varepsilon \rightarrow 0)$. (Мы здесь следуем рассуждениям работы [2], см. также [5]; несколько другой подход к построению асимптотических решений, дающий, однако, те же самые уравнения эволюции медленных модулящий, был предложен в [6]-[8].)

Нетрудно видеть, что подстановка решения (21) в систему (19) дает в нулевом порядке по $\varepsilon$ уравнения

$$
S_{T}^{\alpha} \Phi_{(0) \theta^{\alpha}}^{i}(\theta, X, T)=Q^{i}\left(\mathbf{\Phi}_{(0)}, S_{X}^{\alpha} \mathbf{\Phi}_{(0) \theta^{\alpha}}, \ldots\right),
$$

т.е. при любых $X$ и $T \boldsymbol{\Phi}_{(0)}(\theta, X, T)$ как функция $\theta$ представляет собой одну из функций описанного вьше семейства $\mathscr{M}$, а функции $\varphi(x, t, \varepsilon)=$ $\boldsymbol{\Phi}\left(\theta_{0}+(1 / \varepsilon) \mathbf{S}(\varepsilon x, \varepsilon t), \varepsilon\right)$, получаемые из (21) заменой $X$ и $T$ на $x$ и $t$ соответственно, приближаются при малых $\varepsilon$ к медленно промодулированным $m$-фазным решениям (2).

Из (22) при этом следует

$$
\mathbf{k}(\mathbf{U})=\mathbf{S}_{X}, \quad \boldsymbol{\omega}(\mathbf{U})=\mathbf{S}_{T}
$$

где $\mathbf{U}, \mathbf{k}$ и $\boldsymbol{\omega}$-введенные выше параметры на многообразии $\mathscr{M}$.

Члены при $\varepsilon^{k}, k>0$, дают условия

$$
\left(\hat{\mathbf{L}}_{\mathbf{U}(\mathbf{X}, \mathbf{T}), \theta_{0}(X, T)} \boldsymbol{\Phi}_{(k)}\right)^{i}(\theta, X, T)=f_{k}^{i}\left(\mathbf{\Phi}_{(0)}, \boldsymbol{\Phi}_{(1)}, \ldots, \boldsymbol{\Phi}_{(k-1)}, \mathbf{S}_{X}, \mathbf{S}_{T}, \ldots\right),
$$

где $\hat{\mathbf{L}}_{\mathbf{U}, \theta_{0}}-$ введенньй в (16) линейный дифференциальный по $\theta$ оператор с периодическими (по $\theta$ ) коэффициентами (выражающимися через $\boldsymbol{\Phi}_{(0)}\left(\theta, \theta_{(0)}(X, T), \mathbf{U}(X, T)\right)$ и ее производные по $\left.\theta^{\alpha}\right) ; \quad \mathbf{f}_{k}$ - невязка, зависящая от предыдущих функций $\boldsymbol{\Phi}_{(j)}(\theta, X, T)$ и имеющая порядок $k$, при условии, что функциям $\boldsymbol{\Phi}_{(j)}$ приписывается порядок $j$, функции $\mathbf{S}-$ порядок -1 и при перемножении функций их порядки складываются, а дифференцирование по $T$ и $X$ повьшает порядок на 1.

Система (24) разрешима в классе $2 \pi$-периодических по $\theta$ функций тогда и только тогда, когда при любых $X$ и $T$ вектор $\mathbf{f}_{k}(\theta, X, T)$ ортогонален любому из введенных в (17) левых собственных векторов оператора $\hat{\mathbf{L}}_{\mathbf{U}(X, T), \theta_{0}(X, T)}$, т.е.

$$
\frac{1}{(2 \pi)^{m}} \int_{0}^{2 \pi} \cdots \int_{0}^{2 \pi} \kappa_{i \mathbf{U}(X, T)}^{(s)}\left(\theta+\theta_{0}\right) f_{k}^{i}(\theta, X, T) d^{m} \theta \equiv 0,
$$

что налагает на функцию $\mathbf{f}_{k}(\theta, X, T)$ при каждых $X$ и $T m+g=N-m$ независимых условий соответственно числу левых собственных векторов оператора $\hat{\mathbf{L}}_{\mathbf{U}(X, T), \theta_{0}(X, T)}$. 
Взяв $\boldsymbol{\Phi}_{(0)}$ в виде $\boldsymbol{\Phi}_{(0)}(\theta, X, T)=\boldsymbol{\Phi}_{\text {in }}\left(\theta+\theta_{0}(X, T), \mathbf{U}(X, T)\right)$, нетрудно видеть из приведенных вьше соображений, что в первом порядке по $\varepsilon$ уравнения (24) имеют вид

$$
\begin{aligned}
& \left(\hat{\mathbf{L}}_{\left[\mathbf{U}(X, T), \theta_{0}(X, T)\right]} \boldsymbol{\Phi}_{1}\right)^{i}(\theta)=\sum_{\mathbf{n}} \alpha_{j(\mathbf{n})}^{i}\left(\mathbf{S}_{X}, \boldsymbol{\Phi}_{(0)}, \boldsymbol{\Phi}_{(0) \theta^{\alpha}}, \boldsymbol{\Phi}_{(0) \theta^{\alpha} \theta^{\beta}}, \ldots\right) \\
& \quad \times\left(\boldsymbol{\Phi}_{(0) \mathbf{n} \theta, U^{\nu}}^{j} U_{X}^{\nu}+\boldsymbol{\Phi}_{(0) \mathbf{n} \theta, \theta^{\alpha}}^{j} \theta_{0 X}^{\alpha}\right)+\beta_{\alpha}^{i}\left(\mathbf{S}_{X}, \boldsymbol{\Phi}_{(0)}, \boldsymbol{\Phi}_{(0) \theta^{\alpha}}, \boldsymbol{\Phi}_{(0) \theta^{\alpha} \theta^{\beta}}, \ldots\right) \mathbf{S}_{X X} \\
& \quad-\left(\boldsymbol{\Phi}_{(0) U^{\nu}}^{i} U_{T}^{\nu}+\boldsymbol{\Phi}_{(0) \theta^{\alpha}}^{i} \theta_{0 T}^{\alpha}\right),
\end{aligned}
$$

где $\mathbf{n}=\left(n_{1}, \ldots, n_{m}\right)$ - целочисленный $m$-вектор с неотрицательными компонентами, обозначение $\mathbf{n} \theta$ означает здесь $\left(n_{1} \theta^{1}, \ldots, n_{m} \theta^{m}\right) ; \alpha_{j(\mathbf{n})}^{i}$ и $\beta_{\alpha}^{i}$ - некоторые функции; величины $\mathbf{S}_{X}$ и $\mathbf{S}_{T}$ связаны с параметрами $\mathbf{U}(X)$, соответствуюшими $\boldsymbol{\Phi}_{(0)}(\theta, X)$, соотношениями (23). Таким образом, условие (25) дает на функции $\mathbf{U}(X, T)$ и $\theta_{0}(X, T) N-m$ уравнений вида

$$
A_{\mu}^{\zeta}(\mathbf{U}) U_{T}^{\mu}+B_{\mu}^{\zeta}(\mathbf{U}) U_{X}^{\mu}+A_{\alpha}^{\prime \zeta}(\mathbf{U}) \theta_{0 T}^{\alpha}+B_{\alpha}^{\prime \zeta}(\mathbf{U}) \theta_{0 X}^{\alpha}=0, \quad \zeta=1, \ldots, N-m .
$$

Добавляя к ним $m$ уравнений

$$
k_{T}^{\alpha}=\omega_{X}^{\alpha},
$$

следующих из (23), получим систему $N$ квазилинейных уравнений для $N+m$ функций $U^{\nu}(X, T), \theta_{0}^{\alpha}(X, T)$. При вьполнении условий $(27)$ и $(28)$ функция $\boldsymbol{\Phi}_{(1)}(\theta, X, T)$ находится при любых $X$ и $T$ из дифференциального уравнения по $\theta$ с точностью до линейной комбинации $N-m$ описанных в А) функций, лежащих в ядре оператоpa $\hat{\mathbf{L}}_{\mathbf{U}(X, T), \theta_{0}(X, T)}$ (обозначим их здесь через $\xi_{\mathrm{U}, \theta_{0}}^{(s)}$ ), т.е. с точностью до функции вида $\sum_{s=1}^{N-m} C_{s}(X, T) \boldsymbol{\xi}_{\mathrm{U}(X), \theta_{0}(X)}^{(s)}$.

Значения коэффициентов $C_{s}(X, T)$ при этом находятся из условия разрешимости системы (24) в следующем порядке по $\varepsilon$, и, таким образом, при выполнении условий (27) и (28) имеется процедура последовательного нахождения членов ряда (20), позволяюшая найти требуемые нам функции $\mathbf{\Phi}(\theta, X, T, \varepsilon)$. Функция $\mathbf{S}(X, T)$ при этом восстанавливается по $\mathbf{k}(\mathbf{U})$ и $\boldsymbol{\omega}(\mathbf{U})$.

Лемма 1. При сформулированных выше условиях, т.е. при наличии $N$ локальных трансляционно инвариантных первых интегралов вида (8) таких, что әволюиия соответствующих им плотностей $\mathscr{P}^{\nu}\left(\boldsymbol{\varphi}, \boldsymbol{\varphi}_{x}, \ldots\right)$ в силу (2) имеет вид

$$
\mathscr{P}_{t}^{\nu}=\mathscr{R}_{x}^{\nu}\left(\varphi, \varphi_{x}, \ldots\right)
$$

для некоторых $\mathscr{R}^{\nu}$, система $(27)$ не содержит $\theta_{0}(X, T)$ и налагает условия лишь на $\mathbf{U}(X, T)$, т.е. при этих предположениях члены, содержащие $\theta_{0 X}$ и $\theta_{0 т}$, ортогональны левым собственным векторам оператора $\hat{\mathbf{L}}$ независимо от значений параметров $\mathbf{U}(X, T)$ и $\theta_{0}(X, T)$, и $A_{\mu}^{\prime \zeta} \equiv 0, B_{\mu}^{\prime \zeta} \equiv 0$.

(То же самое выполняется и в методе Уизема для любой из систем (11), не являюшихся нулевым потоком или оператором сдвига по $x$.)

ДокАЗАТЕЛЬСТво. При выполнении условий (27) и (28) сушествует решение (19) в виде асимптотического ряда (20), подстановка которого в уравнения (29) с последуюшим интегрированием по $\theta$ даст в первом порядке по $\varepsilon$

$$
U_{T}^{\nu}=\partial_{X}\left\langle\mathscr{R}^{\nu}\right\rangle(\mathbf{U})
$$


где $\langle\ldots\rangle$ означает усреднение на функциях семейства $\mathscr{M}$, определяемое формулой

$$
\left\langle F\left(\boldsymbol{\varphi}, \boldsymbol{\varphi}_{x}, \ldots\right)\right\rangle(\mathbf{U}) \equiv \frac{1}{(2 \pi)^{m}} \int_{0}^{2 \pi} \cdots \int_{0}^{2 \pi} F\left(\boldsymbol{\Phi}, k^{\alpha} \boldsymbol{\Phi}_{\theta^{\alpha}}, k^{\alpha} k^{\beta} \boldsymbol{\Phi}_{\theta^{\alpha} \theta^{\beta}}, \ldots\right) d^{m} \theta
$$

при $\varphi(x)=\boldsymbol{\Phi}_{\text {in }}\left(\mathbf{k} x+\theta_{0}, \mathbf{U}\right)$.

Таким образом, в силу условий (27), (28) справедлива система (30), представляющая собой $N$ независимых в общем случае уравнений на $\mathbf{U}(X, T)$ того же вида, что и (27), (28), и, следовательно, (30) эквивалентна (27), (28). Поскольку данные рассуждения применимы к любой из систем (11), не являющейся нулевым потоком или оператором сдвига по $x$, это и доказывает лемму. (Заметим, что при наличии дополнительных законов сохранения вида (29) их усреднение дает уравнения, являющиеся следствием (30), при этом независимость системы (30) $N$ уравнений на $N$ параметров $U^{\nu}(X)$ является свойством общего положения.)

Система (30) (или эквивалентная ей (27), (28)), описывающая эволюцию медленной модулящии параметров $\mathbf{U}(X) m$-фазных решений системы $(2)$ называется системой уравнений Уизема.

Система (30) имеет вид

$$
U_{T}^{\nu}=V_{\mu}^{\nu}(\mathbf{U}) U_{X}^{\mu}, \quad \nu, \mu=1, \ldots, N
$$

и относится к эволюционным системам гидродинамического типа (см. [3], [4]). При заданных гладких начальных данных $\mathbf{U}(X)$ система $(32)$ имеет в общем случае гладкое однозначное решение до некоторого момента $T_{0}$, зависяшего от начальных условий, после чего происходит опрокидывание волн. Таким образом, пользоваться решениями уравнений Уизема можно в обшем случае лишь до определенного момента времени, после которого они не являются определенными.

Заметим также, что имеет смысл рассматривать суммы первых нескольких членов ряда (20), поскольку они задают решение исходной системы (2) с точностью до членов более высокого порядка по $\varepsilon$. В частности, имеет смысл рассматривать только первый член ряда (20), задающий эволюцию медленно промодулированных $m$-фазных решений (2) при условии, что выполняются уравнения Уизема на параметры $\mathbf{U}$, поскольку первая поправка по $\varepsilon$ является в этом случае равномерно ограниченной. Мы в данной статье будем рассматривать возникающие асимптотические ряды по $\varepsilon$ именно с этой точки зрения; в частности, мы не будем исследовать областей сходимости таких рядов, поскольку для наших целей будет важно лишь то, что представляемая рядом величина равна сумме его нескольких первых $\left(\right.$ скажем, $k$ ) членов с точностью до величин порядка $O\left(\varepsilon^{k+1}\right)$ при $\varepsilon \rightarrow 0$. То же самое относится и к соотношениям, накладываемым на такие величины: нам будет необходимо выполнение их с точностью до величин более высокого порядка по $\varepsilon^{1}$.

\section{§3. Скобки Пуассона гидродинамического типа}

Среди систем типа (32) особо выделен класс систем, обладающих свойством гамильтоновости относительно скобки вида

$$
\left\{U^{\nu}(X), U^{\mu}(Y)\right\}=g^{\nu \mu}(\mathbf{U}(X)) \delta^{\prime}(X-Y)+b_{\lambda}^{\nu \mu}(\mathbf{U}(X)) U_{X}^{\lambda} \delta(X-Y)
$$

\footnotetext{
${ }^{1}$ Автор благодарит И. М. Кричевера за плодотворные обсуждения этой стороны вопроса.
} 
с локальным гамильтонианом гидродинамического типа

$$
H=\int h(\mathbf{U}(X)) d x
$$

играюшим важную роль при интегрировании таких систем (см. [9]-[11]).

Теория скобок (33) с невырожденными $g^{\nu \mu}(\mathbf{U})$, построенная Б. А. Дубровиным и С. П. Новиковым (см. [3], [4]), тесно связана с римановой геометрией. В частности, из их кососимметричности следует

$$
g^{\nu \mu}=g^{\mu \nu}, \quad b_{\lambda}^{\nu \mu}+b_{\lambda}^{\mu \nu}=\frac{\partial g^{\nu \mu}}{\partial U^{\lambda}}
$$

а из тождества Лейбница вытекает, что при точечных заменах координат $U^{\nu} \rightarrow$ $\widetilde{U}^{\nu}(\mathbf{U})$ функции $g^{\nu \mu}$ преобразуются как метрика с верхними индексами, а величины $\Gamma_{\mu \lambda}^{\nu}=-g_{\mu \tau} b_{\lambda}^{\tau \nu}$, где $g_{\mu \tau} g^{\tau \nu}=\delta_{\mu}^{\nu}$, - как коэффициенты связности, согласованной в силу (35) с метрикой. Тождество Якоби для скобки (33) в случае невырожденности $g^{\nu \mu}$ равносильно симметричности связности $\Gamma_{\mu \lambda}^{\nu}$ и равенству нулю тензора кривизны метрики: $R_{\mu \lambda \tau}^{\nu} \equiv 0$. Теория скобок (33) с вырожденным тензором $g^{\nu \mu}$ более сложна, но также имеет красивую дифференциально-геометрическую интерпретацию (см. [15]).

Б. А. Дубровиным и С.П. Новиковым в [4] был также предложен алгоритм построения скобки вида (33) для системы уравнений Уизема (32), исходя из гамильтоновой структуры (3) для системы (2), а также нужного количества попарно коммутируюших первых интегралов (8). А именно, пусть в силу скобки (3) попарные скобки плотностей интегралов (8) имеют вид

$$
\left\{\mathscr{P}^{\nu}\left(\varphi(x), \varphi_{x}, \ldots\right), \mathscr{P}^{\mu}\left(\varphi(y), \varphi_{y}, \ldots\right)\right\}=\sum_{k \geqslant 0} A_{k}^{\nu \mu}\left(\varphi(x), \varphi_{x}, \ldots\right) \delta^{k}(x-y)
$$

(в сумме присутствует конечное число слагаемых, $\nu, \mu=1, \ldots, N)$.

В силу (9) выполняются соотношения

$$
A_{0}^{\nu \mu}\left(\varphi, \varphi_{x}, \ldots\right) \equiv \partial_{x} Q^{\nu \mu}\left(\varphi, \varphi_{x}, \ldots\right)
$$

для некоторых $Q^{\nu \mu}$. Во введенных выше координатах $\mathbf{U}=\left(U^{1}, \ldots, U^{N}\right)$ скобка Дубровина-Новикова имеет вид

$$
\left\{U^{\nu}(X), U^{\mu}(Y)\right\}=\left\langle A_{1}^{\nu \mu}\right\rangle(\mathbf{U}(X)) \delta^{\prime}(X-Y)+\frac{\partial\left\langle Q^{\nu \mu}\right\rangle(\mathbf{U}(X))}{\partial X} \delta(X-Y),
$$

где $\langle\ldots\rangle$, как и раньше, означает усреднение по $m$-фазным решениям $(2)$, определяемое формулой (31).

В [4], однако, отсутствовало доказательство того, что скобка (38) удовлетворяет при этом тождеству Якоби, о чем сообщалось в [12]. Основная цель данной работы состоит в обосновании того факта, что вышеописанная процедура (36)-(38) усреднения скобки (3) действительно дает скобку Пуассона вида (33), удовлетворяющую тождеству Якоби.

Для дальнейшего нам понадобится процедура ограничения по Дираку скобки Пуассона на подмногообразие. Опишем здесь эту процедуру, используя обозначения конечномерных пространств. 
Пусть в пространстве $V$ с координатами $\mathbf{x}=\left(x^{1}, \ldots, x^{l}\right)$ и скобкой Пуассона

$$
\left\{x^{q}, x^{p}\right\}=J^{q p}(\mathbf{x})
$$

с помощью связей $g^{1}, \ldots, g^{s}$ выделено подмногообразие $W$ :

$$
g^{1}(\mathbf{x})=0, \ldots, g^{s}(\mathbf{x})=0 .
$$

Предположим также, что некоторые $l-s$ функций $f^{1}(\mathbf{x}), \ldots, f^{l-s}(\mathbf{x})$, определенные в некоторой окрестности $W$ в пространстве $V$, при ограничении на $W$ задают на нем систему координат. Любое переопределение функций $f^{\lambda}(\mathbf{x})$ вида

$$
\tilde{f}^{\lambda}(\mathbf{x})=f^{\lambda}(\mathbf{x})+\sum_{\zeta=1}^{s} \tau_{\zeta}^{\lambda}(\mathbf{x}) g^{\zeta}(\mathbf{x})
$$

с произвольными $\tau_{\zeta}^{\lambda}(\mathbf{x})$ не меняет этой системы. Допустим, что функции $\tau_{\zeta}^{\lambda}(\mathbf{x})$ удалось выбрать таким образом, что гамильтонов поток, порождаемый каждой из $\tilde{f}^{\lambda}(\mathbf{x})$, оставляет $W$ инвариантным (в конечномерном случае при условии невырожденности матрищы $\left\{g^{\zeta}(\mathbf{x}), g^{\xi}(\mathbf{x})\right\}$ на подмногообразии $W$ этого всегда можно добиться), т.е. $\left\{\tilde{f}^{\lambda}(\mathbf{x}), g^{\zeta}(\mathbf{x})\right\} \equiv 0$ при $\mathbf{g}(\mathbf{x})=0$. Тогда можно определить ограничение по Дираку скобки (39) на подмногообразие (40), положив на нем

$$
\left\{f^{\lambda}, f^{\mu}\right\}^{*}=\left.\left\{\tilde{f}^{\lambda}(\mathbf{x}), \tilde{f}^{\mu}(\mathbf{x})\right\}\right|_{W}(\mathbf{f}) .
$$

Скобка (41) при этом автоматически удовлетворяет всем необходимым тождествам и называется ограничением по Дираку скобки (39) на подмногообразие (40). Нетрудно проверить, что при найденных таким образом функциях $\tau_{\zeta}^{\lambda}(\mathbf{x})$ скобка (41) будет иметь вид

$$
\left\{f^{\lambda}, f^{\mu}\right\}^{*}=\left.\left\{f^{\lambda}(\mathbf{x}), f^{\mu}(\mathbf{x})\right\}\right|_{W}(\mathbf{f})-\left.\left(\tau_{\zeta}^{\lambda}(\mathbf{x}) \tau_{\xi}^{\mu}(\mathbf{x})\left\{g^{\zeta}(\mathbf{x}), g^{\xi}(\mathbf{x})\right\}\right)\right|_{W}(\mathbf{f}) .
$$

Формула (42), вернее, ее бесконечномерный аналог, будет удобна в наших дальнейших рассуждениях.

\section{§4. Координаты в окрестности подмногообразия, соответствуюшего полному набору $m$-фазных решений}

Вернемся теперь к рассмотрению связанных с методом Уизема конструкций. После замены $X=\varepsilon x$ скобка (3) переходит в

$$
\left\{\varphi^{i}(X), \varphi^{j}(Y)\right\}=\sum_{k \geqslant 0} B_{k}^{i j}\left(\varphi, \varepsilon \varphi_{X}, \varepsilon^{2} \varphi_{X X}, \ldots\right) \varepsilon^{k} \delta^{(k)}(X-Y),
$$

а интегралы (8) приобретают вид

$$
I^{\nu}=\varepsilon^{-1} \int \mathscr{P}\left(\varphi, \varepsilon \varphi_{X}, \ldots\right) d X .
$$

Рассмотрим пространство функций $\varphi\left(\theta^{1}, \ldots, \theta^{m}, X\right), 2 \pi$-периодических по каждому из $\theta^{\alpha}$, и определим на нем при любом $\varepsilon$ скобку Пуассона по формуле

$$
\left\{\varphi^{i}(\theta, X), \varphi^{j}\left(\theta^{\prime}, Y\right)\right\}=\sum_{k \geqslant 0} B_{k}^{i j}\left(\boldsymbol{\varphi}, \varepsilon \varphi_{X}, \varepsilon^{2} \varphi_{X X}, \ldots\right) \varepsilon^{k} \delta^{(k)}(X-Y) \delta\left(\theta-\theta^{\prime}\right)
$$


(под $\delta\left(\theta-\theta^{\prime}\right)$ здесь понимается $\delta$-функция в $m$-мерном пространстве; очевидно, что (45) при любом $\varepsilon$ задает скобку Пуассона).

В пространстве $2 \pi$-периодических по $\theta$ функций рассмотрим подмногообразие $\mathscr{M}^{\prime}$ функций $\varphi(\theta, X)$ таких, что при любом $X \varphi(\theta, X)$ как функция $\theta$ принадлежит $\mathscr{M}$. За координаты на $\mathscr{M}^{\prime}$ можно взять значения $U^{\nu}(X)$ и $\theta_{0}^{\alpha}(X) ;$ функции $\varphi(\theta, X)$, принадлежашие $\mathscr{M}^{\prime}$, будут при этом задаваться формулой

$$
\varphi(\theta, X)=\boldsymbol{\Phi}_{\mathrm{in}}\left(\theta+\theta_{0}(X), \mathbf{U}(X)\right)
$$

После продолжения координат $\mathbf{U}(X)$ и $\theta_{0}(X)$ не зависяшим от $\varepsilon$ образом на некоторую окрестность $\Delta_{\delta}$ подмнообразия $\mathscr{M}^{\prime}$ в функциональном пространстве $2 \pi$-периодических по $\theta$ функций, определяемую, к примеру, условиями

$$
\begin{aligned}
\varphi(\theta, X) \in \Delta_{\delta} \Leftrightarrow \exists \varphi_{0}(\theta, X) \in \mathscr{M}^{\prime}:\left\|\varphi(\theta, X)-\varphi_{0}(\theta, X)\right\| \\
\equiv \sum_{i}\left(\max \left|\varphi^{i}(\theta, X)-\varphi_{0}^{i}(\theta, X)\right|\right. \\
+\frac{1}{1 !}\left(\sum_{\alpha} \max \left|\varphi_{\theta^{\alpha}}^{i}-\varphi_{0 \theta^{\alpha}}^{i}\right|+\max \left|\varphi_{X}^{i}-\varphi_{0 X}^{i}\right|\right) \\
+\frac{1}{2 !}\left(\sum_{\alpha, \beta} \max \left|\varphi_{\theta^{\alpha} \theta^{\beta}}^{i}-\varphi_{0 \theta^{\alpha}, \theta^{\beta}}^{i}\right|+\sum_{\alpha} \max \left|\varphi_{\theta^{\alpha} X}^{i}-\varphi_{0 \theta^{\alpha} X}^{i}\right|\right. \\
\left.\left.+\max \left|\varphi_{X X}^{i}-\varphi_{0 X X}^{i}\right|\right)+\frac{1}{3 !}(\ldots)+\ldots\right)<\delta
\end{aligned}
$$

(т.е. подразумевается, что существует функция $\varphi_{0}(\theta, X)$, принадлежащая $\mathscr{M}^{\prime}$, такая, что все члены ряда (47) сушествуют, ряд (47) сходится и удовлетворяет написанному условию), подмногообразие $\mathscr{M}^{\prime}$ можно задать с помошью системы связей

$$
F^{i}(\theta, X)[\boldsymbol{\Phi}]=\omega^{\alpha}(\mathbf{U}[\boldsymbol{\varphi}](X)) \varphi_{\theta^{\alpha}}^{i}-Q^{i}\left(\boldsymbol{\varphi}, k^{\alpha}(\mathbf{U}[\varphi](X)) \varphi_{\theta^{\alpha}}, \ldots\right)=0 .
$$

От системы (6) система (48) отличается тем, что она уже не зависит от $\mathbf{k}$ и $\boldsymbol{\omega}$, поскольку здесь они являются вполне определенными функционалами $\varphi(\theta, X)$ (в окрестности $\left.\mathscr{M}^{\prime}\right)$, значения которых на $\mathscr{M}^{\prime}$ совпадают с соответствуюшими параметрами семейства. Нетрудно видеть, что системе (48) при этом удовлетворяют функции из $\mathscr{M}^{\prime}$ и только они.

Функционалы $\mathbf{U}(X)$ и $\theta_{0}(X)$ в окрестности $\Delta_{\delta}$ можно ввести, например, следующим образом: введем в $\Delta_{\delta} N$ каких-нибудь функционалов вида

$$
a^{\nu}[\varphi](X)=\frac{1}{(2 \pi)^{m}} \int_{0}^{2 \pi} \cdots \int_{0}^{2 \pi} A^{\nu}\left(\varphi(\theta, X), \varphi_{\theta^{\alpha}}(\theta, X), \ldots\right) d^{m} \theta
$$

с некоторыми функциями $A^{\nu}$ такими, что на $\mathscr{M}^{\prime}$ значения $a^{\nu}$ функционально независимы. На $\mathscr{M}^{\prime}$ значения $a^{\nu}$, как нетрудно видеть, выражаются через $\mathbf{U}(X)$ и не зависят от $\theta_{0}(X)$. Таким образом, разбив $\mathscr{M}^{\prime}$ на "карты", в каждой из которых $\left|U_{(1)}^{\nu}(X)-U_{(2)}^{\nu}(X)\right|<\delta^{\prime}$, можно в каждой из таких карт выразить $\mathbf{U}(X)$ через $a^{\nu}(X)$ в виде $U^{\nu}(X)=f^{\nu}(\mathbf{a}(X))$ и затем, используя определение (49), продолжить по этим формулам $U^{\nu}(X)$ в окрестности каждой из карт (это может быть 
сделано независимо при каждом $X)$. Затем, определив таким образом функционалы $U^{\nu}(X)$ в окрестности $\Delta_{\delta}$, рассмотрим в $\Delta_{\delta}$ функционалы

$$
\vartheta^{\alpha}(X)=\frac{1}{(2 \pi)^{m}} \int_{0}^{2 \pi} \cdots \int_{0}^{2 \pi} \sum_{i} \varphi^{i}(\theta, X) \Phi_{\operatorname{in} \theta^{\alpha}}^{i}(\theta, \mathbf{U}[\varphi](X)) d^{m} \theta,
$$

где $\boldsymbol{\Phi}_{\text {in }}(\theta, \mathbf{U})$ - введенные в (46) функции из $\mathscr{M}^{\prime}$.

Нетрудно видеть, что при $\varphi(\theta, X) \in \mathscr{M}^{\prime}$ и $\theta_{0}(X) \equiv 0$ выполняются соотношения $\vartheta^{\alpha}(X) \equiv 0$, и в случае общего положения при равномерно малых $\theta_{0}^{\alpha}(X)$ можно однозначно выразить $\left\{\theta_{0}^{\alpha}(X)\right\}$ через $\left\{\vartheta^{\alpha}(X)\right\}$ на $\mathscr{M}^{\prime}$ в виде $\theta_{0}^{\alpha}(X)=\tau^{\alpha}(\boldsymbol{\vartheta}(X))$. Аналогично, разбивая, если нужно, каждую из вьшеописанных карт дополнительно на карты, в каждой из которых $\left|\theta_{0(1)}^{\alpha}(X)-\theta_{0(2)}^{\alpha}(X)\right|<\delta^{\prime \prime}$ (независимо при каждом $X)$, и выражая такими формулами $\theta_{0}^{\alpha}(X)$ через $\vartheta(X)$, можно продолжить $\theta_{0}^{\alpha}(X)$ в $\Delta_{\delta}$, пользуясь функционалами $\vartheta(X)$.

Нетрудно получить, что при таком определении $\mathbf{U}(X)$ и $\theta_{0}(X)$ в $\Delta_{\delta}$ для двух функций $\varphi_{(1)}(\theta, X)$ и $\varphi_{(2)}(\theta, X)$ из $\Delta_{\delta}$, удовлетворяюших соотношению типа (47), т.е. $\left\|\varphi_{(1)}(\theta, X)-\varphi_{(2)}(\theta, X)\right\|<\delta$, где $\delta$ мало, выполняются условия

$$
\left|U_{(1)}^{\nu}(X)-U_{(2)}^{\nu}(X)\right|<C \delta, \quad\left|\theta_{0(1)}^{\alpha}(X)-\theta_{0(2)}^{\alpha}(X)\right|<C \delta,
$$

где $C$-некоторая константа. Аналогичные соотношения будут вьполняться и для вариационных производных $\mathbf{U}(X)$ и $\theta_{0}(X)$.

Мы будем предполагать, что после такого продолжения подмногообразие $\mathscr{M}^{\prime}$, задаваемое системой (48), обладает свойством регулярности, аналогичным регулярности $\mathscr{M}_{\boldsymbol{\omega}, \mathbf{k}}$. Ясно, что перечисленные ранее векторы

$$
\boldsymbol{\Phi}_{\theta^{\alpha}}\left(\theta+\theta_{0}(X), \mathbf{U}(X)\right), \quad \boldsymbol{\Phi}_{U^{\nu}}\left(\theta+\theta_{0}(X), \mathbf{U}(X)\right)
$$

(обозначим этот набор через $\left\{\tilde{\boldsymbol{\xi}}_{\left[\mathbf{U}, \theta_{0}\right]}^{(q)}(X), q=1, \ldots, N+m\right\}$ ) при каждом значении $X$ лежат в ядре линеаризованного на функции из $\mathscr{M}^{\prime}$ функционала $F^{i}(\theta, X)[\varphi]$, введенного в (48):

$$
\begin{aligned}
& \delta F^{i}(\theta, X)=\frac{1}{(2 \pi)^{m}} \int_{0}^{2 \pi} \cdots \int_{0}^{2 \pi} \tilde{L}_{j[\mathbf{U}]}^{i}\left(\theta+\theta_{0}(X), \theta^{\prime}+\theta_{0}(X), X\right) \delta \varphi^{j}\left(\theta^{\prime}, X\right) d^{m} \theta^{\prime} \\
& =\frac{1}{(2 \pi)^{m}} \int_{0}^{2 \pi} \cdots \int_{0}^{2 \pi}\left[\left(\omega^{\alpha}(\mathbf{U}(X)) \delta_{j}^{i} \delta_{\theta^{\alpha}}\left(\theta-\theta^{\prime}\right)-\left.\frac{\partial Q^{i}}{\partial \varphi^{j}}\right|_{\mathbf{k}=\mathrm{const}} \delta\left(\theta-\theta^{\prime}\right)\right.\right. \\
& \left.\quad-\left.\frac{\partial Q^{i}}{\partial \varphi_{\theta^{\alpha}}^{j}}\right|_{\mathbf{k}=\mathrm{const}} \delta_{\theta^{\alpha}}\left(\theta-\theta^{\prime}\right)-\left.\frac{\partial Q^{i}}{\partial \varphi_{\theta^{\alpha} \theta^{\beta}}^{j}}\right|_{\mathbf{k}=\mathrm{const}} \delta_{\theta^{\alpha} \theta^{\beta}}\left(\theta-\theta^{\prime}\right)-\ldots\right) \\
& \left.\quad+\left(\frac{\partial \omega^{\alpha}}{\partial U^{\nu}}(X) \frac{\delta U^{\nu}(X)}{\delta \varphi^{j}\left(\theta^{\prime}, X\right)} \varphi_{\theta^{\alpha}}^{i}(\theta, X)-\frac{\partial Q^{i}}{\partial U^{\nu}}(\theta, X) \frac{\delta U^{\nu}(X)}{\delta \varphi^{j}\left(\theta^{\prime}, X\right)}\right)\right] \delta \varphi^{j}\left(\theta^{\prime}, X\right) d^{m} \theta^{\prime} .
\end{aligned}
$$

Будем предполагать, что других векторов, обладающих этим свойством, нет, и, кроме того, в каждой точке $\mathscr{M}^{\prime}$ существует при каждом $X$ ровно $N+m$ "левых собственных векторов" $\tilde{\boldsymbol{\kappa}}_{\left[\mathbf{U}, \theta_{0}\right]}^{(q)}(X)$ для оператора $\hat{\tilde{\mathbf{L}}}_{\left[\mathbf{U}, \theta_{0}\right]}$ с нулевыми собственными значениями, т.е. таких линейно независимых $2 \pi$-периодических функций $\tilde{\kappa}_{i[\mathbf{U}]}^{(q)}\left(\theta+\theta_{0}, X\right), i=1, \ldots, n$, что

$$
\frac{1}{(2 \pi)^{m}} \int_{0}^{2 \pi} \cdots \int_{0}^{2 \pi} \tilde{\kappa}_{i[\mathbf{U}]}^{(q)}(\theta, X) \tilde{L}_{j[\mathbf{U}]}^{i}\left(\theta, \theta^{\prime}, X\right) d^{m} \theta \equiv 0 .
$$


Заметим, что, в отличие от $\left\{\tilde{\boldsymbol{\xi}}_{\left[\mathbf{U}, \theta_{0}\right]}^{(q)}(X)\right\}$, вид функций $\left\{\tilde{\boldsymbol{\kappa}}_{\left[\mathbf{U}, \theta_{0}\right]}^{(q)}(X)\right\}$ зависит от способа продолжения $\mathbf{U}(X)$ и $\theta_{0}(X)$ в $\Delta_{\delta}$, поскольку $\tilde{\boldsymbol{\xi}}^{(q)}$, лежащие в ядре $\hat{\tilde{\mathbf{L}}}$, соответствуют вариациям функций $\varphi(\theta, X)$ вдоль $\mathscr{M}^{\prime}$, на которых $\mathbf{U}(X)$ и $\theta_{0}(X)$ заданы однозначно, в то время как $\tilde{\boldsymbol{\kappa}}^{(q)}(\theta, X)$ связаны с образом оператора $\hat{\tilde{\mathbf{L}}}$, для нахождения которого необходимо знать изменение $\mathbf{U}(X)$ и $\theta_{0}(X)$ при всевозможных вариациях $\varphi(\theta, X)$.

С помощью описанных выше конструкций в $\Delta_{\delta}$ можно вместо “стандартной" системы координат, образованной функционалами $\varphi^{i}(\theta, X)$, ввести другую систему, приняв за координаты в $\Delta_{\delta}$ функционалы $\left\{U^{\nu}(X), \theta_{0}^{\alpha}(X), \nu=1, \ldots, N\right.$, $\alpha=1, \ldots, m\}$, а также величины

$$
\begin{aligned}
G_{\left[\mathbf{U}, \theta_{0}\right]}^{i}[\varphi](\theta, X)= & \frac{1}{(2 \pi)^{m}} \int_{0}^{2 \pi} \cdots \int_{0}^{2 \pi} \tilde{L}_{j[\mathbf{U}]}^{i}\left(\theta+\theta_{0}(X), \theta^{\prime}+\theta_{0}(X), X\right) \\
& \times\left(\varphi^{j}\left(\theta^{\prime}, X\right)-\Phi_{\mathrm{in}}^{j}\left(\theta^{\prime}+\theta_{0}(X), \mathbf{U}(X)\right) d^{m} \theta^{\prime},\right.
\end{aligned}
$$

где $\hat{\tilde{L}}_{j[\mathrm{U}]}^{i}$ функционалы из (51), возникающие при линеаризации связей $F^{i}(\theta, X)$ на подмногообразии $\mathscr{M}^{\prime}$.

Функции $\varphi(\theta, X)$ из $\Delta_{\delta}$ будут, таким образом, характеризоваться набором $N+m$ гладких функций $\mathbf{U}^{\nu}(X), \theta_{0}^{\alpha}(X)$, а также функциями $G^{i}(\theta, X), i=1, \ldots, n$, принимающими значения в пространстве $2 \pi$-периодических по $\theta$ функций, удовлетворяющих при каждом значении $X$ и заданных $\mathbf{U}(X)$ и $\theta_{0}(X) N+m$ линейным интегральным условиям вида

$$
\frac{1}{(2 \pi)^{m}} \int_{0}^{2 \pi} \cdots \int_{0}^{2 \pi} \tilde{\kappa}_{i[\mathbf{U}]}^{(q)}\left(\theta+\theta_{0}(X), X\right) G^{i}(\theta, X) d^{m} \theta=0
$$

(Условия такого типа можно встретить, например, в теории расслоений.)

ЛЕмма 2. При достаточно мальх $\delta$ в окрестности $\Delta_{\delta}$ по значениям функиионалов $\mathbf{U}^{\nu}(X), \theta_{0}^{\alpha}(X) u G^{i}(\theta, X)$, удовлетворяющим $(54)$, значения $\varphi^{i}(\theta, X)$ восстанавливаются однозначно.

ДокАЗАТЕЛЬСтво. Действительно, система

$$
\begin{aligned}
\frac{1}{(2 \pi)^{m}} & \int_{0}^{2 \pi} \cdots \int_{0}^{2 \pi} \tilde{L}_{j[\mathbf{U}]}^{i}\left(\theta+\theta_{0}(X), \theta^{\prime}+\theta_{0}(X), X\right) \\
& \times\left(\varphi^{j}\left(\theta^{\prime}, X\right)-\Phi_{\mathrm{in}}^{j}\left(\theta^{\prime}+\theta_{0}(X), \mathbf{U}(X)\right) d^{m} \theta^{\prime m}=G^{i}(\theta, X)\right.
\end{aligned}
$$

имеет при выполнении условия (54) решение, определяемое с точностью до линейной комбинации описанных выше векторов $\left\{\boldsymbol{\xi}_{\left[\mathbf{U}, \theta_{0}\right]}^{(q)}(X)\right\}$, касательных к $\mathscr{M}^{\prime}$ и соответствуюших изменениям параметров $\mathbf{U}(X)$ и $\theta_{0}^{\alpha}(X)$. Таким образом, коэффициенты при них в достаточно малой окрестности $\Delta_{\delta}$ задаются однозначно значениями $U^{\nu}(X)$ и $\theta_{0}^{\alpha}(X)$. Лемма доказана.

Нам понадобится также другая система координат в окрестности $\mathscr{M}^{\prime}$, получаю-

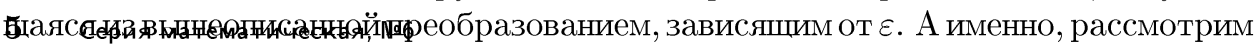


функционалы

$$
\begin{gathered}
J^{\nu}(X)=\frac{1}{(2 \pi)^{m}} \int_{0}^{2 \pi} \cdots \int_{0}^{2 \pi} \mathscr{P}^{\nu}\left(\varphi(\theta, X), \varepsilon \varphi_{X}(\theta, X), \ldots\right) d^{m} \theta, \quad \nu=1, \ldots, N \\
\theta_{0}^{*}(X)=\theta_{0}(X)-\frac{1}{\varepsilon} \int_{X_{0}}^{X} \mathbf{k}\left(\mathbf{J}\left(X^{\prime}\right)\right) d X^{\prime}
\end{gathered}
$$

(для некоторой начальной точки $X_{0}$ ) и за систему координат в окрестности $\mathscr{M}^{\prime}$ возьмем совокупность функционалов $J^{\nu}(X), \theta_{0}^{*}(X)$, а также связей $G^{i}(\theta, X)$. Переход от координат $\left(U^{\nu}(X), \theta_{0}^{\alpha}(X)\right)$ к $\left(J^{\nu}(X), \theta_{0}^{* \alpha}(X)\right)$, как видно из (56), определен при $\varepsilon \neq 0$. При этом непосредственно на многообразии $\mathscr{M}^{\prime}$ (т.е. при $\left.G^{q}(\theta, X) \equiv 0\right)$ при $\varepsilon \rightarrow 0$

$$
\begin{aligned}
& J^{\nu}(X)= \frac{1}{(2 \pi)^{m}} \int_{0}^{2 \pi} \cdots \int_{0}^{2 \pi} \mathscr{P}^{\nu}\left(\boldsymbol{\Phi}_{\mathrm{in}}\left(\theta+\theta_{0}(X), \mathbf{U}(X)\right),\right. \\
&\left.\varepsilon\left(\theta_{0 X}^{\alpha} \boldsymbol{\Phi}_{\mathrm{in} \theta^{\alpha}}+U_{X}^{\nu} \boldsymbol{\Phi}_{\mathrm{in} U^{\nu}}\right), \ldots\right) d^{m} \theta \\
&=\sum_{k \geqslant 0} \varepsilon^{k} J_{(k)}^{\nu}\left(\mathbf{U}, \mathbf{U}_{X}, \ldots, \mathbf{U}_{k X}, \theta_{0 X}, \ldots, \theta_{0 k X}\right),
\end{aligned}
$$

где $J_{(k)}^{\nu}$ представляет собой усредненный по $\theta \quad k$-й тейлоровский член разложения $\mathscr{P}^{\nu}$ при $\varepsilon \rightarrow 0$ на функциях из $\mathscr{M}^{\prime}$. В сумме присутствует конечное число членов, введены обозначения

$$
\mathbf{U}_{k X} \equiv \frac{\partial^{k} \mathbf{U}}{\partial X^{k}}, \quad \theta_{0 k X} \equiv \frac{\partial^{k} \theta_{0}}{\partial X^{k}} .
$$

Выражение для $\theta_{0}^{*}(X)$ через $\mathbf{U}(X)$ и $\theta_{0}(X)$ на $\mathscr{M}^{\prime}$ можно получить, подставляя полученное разложение для $\mathbf{J}(X)$ в $(56)$.

В дальнейшем нам понадобится также обратный переход от $\mathbf{J}(X), \theta_{0}^{*}(X)$ к $\mathbf{U}(X)$, $\theta_{0}(X)$ при $G^{q}(\theta, X)$ (на $\left.\mathscr{M}^{\prime}\right)$. Для получения его заметим, что величины $J^{\nu}(X), \theta_{0}^{* \alpha}$ и $U^{\mu}(X)$ связаны на $\mathscr{M}^{\prime}$ соотношениями (определение $J^{\nu}(X)$ )

$$
\begin{aligned}
& J^{\nu}(X)= \frac{1}{(2 \pi)^{m}} \int_{0}^{2 \pi} \cdots \int_{0}^{2 \pi} \mathscr{P}^{\nu}\left(\boldsymbol{\Phi}_{\text {in }}\left(\theta+\theta_{0}^{*}(X)+\frac{1}{\varepsilon} \int_{X_{0}}^{X} \mathbf{k}\left(\mathbf{J}\left(X^{\prime}\right)\right) d X^{\prime}, \mathbf{U}(X)\right),\right. \\
&\left.\varepsilon \partial_{X} \boldsymbol{\Phi}_{\text {in }}\left(\theta+\theta_{0}^{*}(X)+\frac{1}{\varepsilon} \int_{X_{0}}^{X} \mathbf{k}\left(\mathbf{J}\left(X^{\prime}\right)\right) d X^{\prime}, \mathbf{U}(X)\right), \ldots\right) d^{m} \theta \\
&=\frac{1}{(2 \pi)^{m}} \int_{0}^{2 \pi} \cdots \int_{0}^{2 \pi} \mathscr{P}^{\nu}\left(\boldsymbol{\Phi}_{\text {in }}\left(\theta+\theta_{0}^{*}(X)+\frac{1}{\varepsilon} \int_{X_{0}}^{X} \mathbf{k}\left(\mathbf{J}\left(X^{\prime}\right)\right) d X^{\prime}, \mathbf{U}(X)\right),\right. \\
&\left.\quad k^{\alpha}(\mathbf{J}) \partial_{\theta^{\alpha}} \boldsymbol{\Phi}_{\text {in }}\left(\theta+\theta_{0}^{*}(X)+\frac{1}{\varepsilon} \int_{X_{0}}^{X} \mathbf{k}\left(\mathbf{J}\left(X^{\prime}\right)\right) d X^{\prime}, \mathbf{U}(X)\right), \ldots\right) d^{m} \theta \\
&+\sum_{k \geqslant 1} \varepsilon^{k} \frac{1}{(2 \pi)^{m}} \int_{0}^{2 \pi} \cdots \int_{0}^{2 \pi} \mathscr{P}_{(k)}^{\nu}\left(\boldsymbol{\Phi}_{\text {in }}(\ldots), \ldots\right) d^{m} \theta .
\end{aligned}
$$

Здесь $\mathscr{P}_{(k)}^{\nu}\left(\boldsymbol{\Phi}_{\text {in }}(\ldots), \ldots\right)$ - локальные функционалы, зависяшие от

$$
\boldsymbol{\Phi}_{\text {in }}\left(\theta+\theta_{0}^{*}(X)+\frac{1}{\varepsilon} \int_{X_{0}}^{X} \mathbf{k}\left(\mathbf{J}\left(X^{\prime}\right)\right) d X^{\prime}, \mathbf{U}(X)\right),
$$


и от их производных по $U^{\nu}$ и $\theta^{\alpha}$ с коэффициентами типа $\mathbf{U}_{X}(X), \mathbf{U}_{X X}(X), \ldots$ $\ldots, \mathbf{k}(\mathbf{J}), \partial_{X} \mathbf{k}(\mathbf{J}), \partial_{X}^{2} \mathbf{k}(\mathbf{J}), \ldots$ и $\theta_{0 X}^{*}(X), \theta_{0 X X}^{*}(X), \ldots$ и получающиеся при собирании вместе таких членов, имеющих общий множитель $\varepsilon^{k}$. Отдельно выделен член, соответствующий нулевой степени $\varepsilon$.

После интегрирования по $\theta$, устраняющего нерегулярный при $\varepsilon \rightarrow 0$ сдвиг фаз $\theta_{0}$ в аргументе $\boldsymbol{\Phi}_{\text {in }}$, получим на $\mathscr{M}^{\prime}$

$$
J^{\nu}(X)=\zeta^{\nu}(\mathbf{J}, \mathbf{U})+\sum_{k \geqslant 1} \varepsilon^{k} \zeta_{(k)}^{\nu}\left(\mathbf{U}, \mathbf{U}_{X}, \ldots, \mathbf{U}_{k X}, \mathbf{J}, \mathbf{J}_{X}, \ldots, \mathbf{J}_{k X}, \theta_{0 X}^{*}, \ldots, \theta_{0 k X}^{*}\right)
$$

Сумма (57) содержит конечное число членов, функции $\zeta_{(k)}^{\nu}$ и $\zeta^{\nu}$ есть проинтегрированные по $\theta$ функции $\mathscr{P}_{(k)}^{\nu}$ и $\mathscr{P}^{\nu}$ соответственно. При этом, так как

$$
\begin{aligned}
\zeta^{\nu}(\mathbf{J}, \mathbf{U})= & \frac{1}{(2 \pi)^{m}} \int_{0}^{2 \pi} \cdots \int_{0}^{2 \pi} \mathscr{P}^{\nu}\left(\boldsymbol{\Phi}_{\mathrm{in}}(\theta, \mathbf{U}(X)), k^{\alpha}(\mathbf{J}) \partial_{\theta^{\alpha}} \boldsymbol{\Phi}_{\mathrm{in}}(\theta, \mathbf{U}(X)),\right. \\
& \left.k^{\alpha}(\mathbf{J}) k^{\beta}(\mathbf{J}) \partial_{\theta^{\alpha}} \partial_{\theta^{\beta}} \boldsymbol{\Phi}_{\mathrm{in}}(\theta, \mathbf{U}(X)), \ldots\right) d^{m} \theta
\end{aligned}
$$

(здесь выброшен несущественный при интегрировании по $\theta$ сдвиг начальной фазы в $\left.\boldsymbol{\Phi}_{\text {in }}\right)$, нетрудно видеть, что системе уравнений

$$
J^{\nu}(X)=\zeta^{\nu}(\mathbf{J}(X), \mathbf{U}(X))
$$

удовлетворяют решения $J^{\nu}(X) \equiv U^{\nu}(X)$ согласно определению (10) параметров $\mathbf{U}(X)$ на семействе $\mathscr{M}^{\prime}$. Считая систему (58) системой общего положения, мы будем полагать, что она эквивалентна системе $J^{\nu}(X)=U^{\nu}(X)$.

Учитьвая сделанное замечание, систему (57) можно однозначно разрешить методом последовательных приближений, полагая в нулевом приближении $U^{\nu}(X)=$ $J^{\nu}(X)$; для $U^{\nu}(X)$ при этом получится выражение вида

$$
U^{\nu}(X)=J^{\nu}(X)+\sum_{k \geqslant 1} \varepsilon^{k} U_{(k)}^{\nu}\left(\mathbf{J}, \mathbf{J}_{X}, \ldots, \mathbf{J}_{k X}, \theta_{0 X}^{*}, \ldots, \theta_{0 k X}^{*}\right) .
$$

Подстановка (59) в (57) при условии невырожденности детерминанта $\left.\left\|\frac{\partial \zeta^{\nu}(\mathbf{J}, \mathbf{U})}{\partial U^{\mu}}\right\|\right|_{\mathbf{U}=\mathbf{J}}$ однозначно определит функции $U_{(k)}^{\nu}$. Величина $\theta_{0}(X)$ выражается через $\mathbf{J}(X)$ и $\theta_{0}^{*}(X)$ по формуле $(56)$.

Нам на самом деле не потребуется знать явно члены ряда (59), за исключением первого.

В дальнейшем выражения (56) и (59) будем записьвать в виде $\theta_{0}^{\alpha}\left[\mathbf{J}, \theta_{0}^{*}, \varepsilon\right]$ и $U^{\nu}\left[\mathbf{J}, \theta_{0}^{*}, \varepsilon\right]$ (не обязательно на $\mathscr{M}^{\prime}$ ). При этом для наших целей, как уже упоминалось ранее, нам будет достаточно существования лишь первых нескольких членов (одного или двух) таких выражений, так же как и достаточно того, чтобы существовало ограничение по Дираку исходной скобки Пуассона на подмногообразие, соответствуюшее набору $m$-фазных решений (2), удовлетворяющее тождеству Якоби с точностью до членов порядка $\varepsilon^{3}$. Мы здесь, однако, сохраним форму записи (59), поскольку процедура нахождения членов таких рядов одинакова во всех порядках по $\varepsilon$. 
Функционалы (55) являются хорошо определенными на всем пространстве функций $\varphi(\theta, X)$, причем гамильтоновы потоки, порождаемые в силу скобки (45) интегралами вида

$$
\int q(X) J^{\nu}(X) d X=\int q(X) \frac{1}{(2 \pi)^{m}} \int_{0}^{2 \pi} \cdots \int_{0}^{2 \pi} \mathscr{P}^{\nu}\left(\boldsymbol{\varphi}, \varepsilon \boldsymbol{\varphi}_{X}, \ldots\right) d^{m} \theta,
$$

имеют для любой гладкой функции $q(X)$ вид

$$
\varphi_{t}^{i}=q(X) Q_{\nu}^{i}\left(\varphi, \varepsilon \varphi_{X}, \ldots\right)+\varepsilon q_{X} \widetilde{Q}_{\nu}^{i}\left(\varphi, \varepsilon \varphi_{X}, \ldots\right)+\varepsilon^{2} q_{X X} \widetilde{\widetilde{Q}}_{\nu}^{i}\left(\varphi, \varepsilon \varphi_{X}, \ldots\right)+\ldots
$$

где $Q_{\nu}^{i}$ - введенные в (11) потоки, порождаемые функционалами $I^{\nu}$, а $\widetilde{Q}_{\nu}^{i}, \widetilde{\widetilde{Q}}_{\nu}^{i}, \ldots-$ некоторые функции. (Производные $q(X)$ по $X$ возникают при взятии вариационной производной, а также в результате применения скобки (45). Легко видеть при этом, что каждое дифференцирование $q(X)$ по $X$ входит с множителем $\varepsilon$.)

\section{§5. Ограничение по Дираку скобки Пуассона на подмногообразие, соответствующее полному набору $m$-фазных решений}

Для ограничения по Дираку скобки (45) на подмногобразие $\mathscr{M}^{\prime}$ в координатах $\mathbf{J}(X), \theta_{0}^{*}(X)$ и $\mathbf{G}^{*}(\theta, X)=\left(G^{i}(\theta, X)\right)$ нам понадобятся их скобки Пуассона между собой (на $\left.\mathscr{M}^{\prime}\right)$; к вычислению этих скобок мы сейчас и перейдем.

Начнем со скобок Пуассона вида $\left\{J^{\nu}(X), J^{\mu}(Y)\right\}$. Нетрудно видеть, что такие скобки в силу (36) имеют вид

$$
\begin{aligned}
& \left\{J^{\nu}(X), J^{\mu}(Y)\right\}= \\
& =\frac{1}{(2 \pi)^{2 m}} \int_{0}^{2 \pi} \cdots \int_{0}^{2 \pi} \sum_{k \geqslant 0} A_{k}^{\nu \mu}\left(\varphi, \varepsilon \varphi_{X}, \ldots\right) \varepsilon^{k} \delta^{(k)}(X-Y) \delta\left(\theta-\theta^{\prime}\right) d^{m} \theta d^{m} \theta^{\prime} \\
& =\frac{1}{(2 \pi)^{m}} \int_{0}^{2 \pi} \cdots \int_{0}^{2 \pi} \sum_{k \geqslant 0} A_{k}^{\nu \mu}\left(\varphi, \varepsilon \varphi_{X}, \ldots\right) \varepsilon^{k} \delta^{(k)}(X-Y) d^{m} \theta
\end{aligned}
$$

при этом в силу (37)

$$
A_{0}^{\nu \mu}\left(\varphi, \varepsilon \varphi_{X}, \varepsilon^{2} \varphi_{X X}, \ldots\right) \equiv \varepsilon \partial_{X} Q^{\nu \mu}\left(\varphi, \varepsilon \varphi_{X}, \varepsilon^{2} \varphi_{X X}, \ldots\right) .
$$

В точках многообразия $\mathscr{M}^{\prime}$ функция $\varphi(\theta, X)$ имеет вид

$$
\varphi^{i}(\theta, X)=\Phi_{\text {in }}^{i}\left(\theta+\theta_{0}^{*}(X)+\frac{1}{\varepsilon} \int_{X_{0}}^{X} \mathbf{k}\left(\mathbf{J}\left(X^{\prime}\right)\right) d X^{\prime}, \mathbf{U}\left[\mathbf{J}, \theta_{0}^{*}, \varepsilon\right](X)\right),
$$

и после подстановки ее в (62) можно получить выражение скобки $\left\{J^{\nu}(X), J^{\mu}(Y)\right\}$ в точках $\mathscr{M}^{\prime}$ с координатами $\left(\mathbf{J}(X), \theta_{0}^{*}(X)\right)$ в виде регулярного при $\varepsilon \rightarrow 0$ ряда по $\varepsilon$ ввиду того, что интегрирование по $\theta$ устраняет особенность при $\varepsilon \rightarrow 0$, присутствуюшую в аргументе функции $\boldsymbol{\Phi}_{\text {in. }}$. Нетрудно видеть, что в силу (63) нулевой член в этом разложении отсутствует, и оно имеет вид

$$
\begin{gathered}
\left.\left\{J^{\nu}(X), J^{\mu}(Y)\right\}\right|_{\mathbf{G}(\theta, X)=0}=\varepsilon\left(\left\langle A_{1}^{\nu \mu}\right\rangle(\mathbf{J}(X)) \delta^{\prime}(X-Y)\right. \\
\left.+\frac{\partial\left\langle Q^{\nu \mu}\right\rangle(\mathbf{J}(X))}{\partial X} \delta(X-Y)\right)+\varepsilon^{2} \mathscr{J}^{\nu \mu}\left[\mathbf{J}, \theta_{0}^{*}, \varepsilon\right]
\end{gathered}
$$


где $\mathscr{J}^{\nu \mu}$ - регулярные при $\varepsilon \rightarrow 0$ функционалы $\mathbf{J}$ и $\theta_{0}^{*}$. Функционалы такого типа, возникающие также и далее, имеют вид регулярных при $\varepsilon \rightarrow 0$ асимптотических рядов по степеням $\varepsilon$ вида

$$
C\left[\mathbf{J}, \theta_{0}^{*}, \varepsilon\right]=\sum_{k \geqslant 0} C_{(k)}\left(\mathbf{J}, \mathbf{J}_{X}, \ldots, \mathbf{J}_{k X}, \theta_{0 X}^{*}, \ldots, \theta_{0 k X}^{*}\right) \varepsilon^{k} .
$$

Здесь использован вид разложения (59), выражающего $\mathbf{U}(X)$ через $\mathbf{J}(X)$ и $\theta_{0}^{*}(X)$ в точках $\mathscr{M}^{\prime}$, согласно которому аргументы $U^{\nu}(X)$ (от которых зависят средние типа $\left\langle A_{1}^{\nu \mu}\right\rangle$ и $\left\langle Q^{\nu \mu}\right\rangle$ на $\mathscr{M}^{\prime}$ ) заменены с точностью до членов более высокого порядка по $\varepsilon$ на $J^{\nu}(X)$. Символ $\langle\ldots\rangle$, как и раньше, означает усреднение на семействе $m$-фазных решений (2), определяемое формулой (31).

Эволюция плотностей законов сохранения $\mathscr{P} \nu\left(\varphi, \varepsilon \varphi_{X}, \ldots\right)$ в силу системы $(61)$, порождаемой функционалом $\int q(X) J^{\mu}(X) d X$, имеет, как нетрудно видеть из $(36)$, вид

$$
\mathscr{P}_{\tau^{\mu}}^{\nu}(X)=\sum_{k \geqslant 0} A_{k}^{\nu \mu}\left(\varphi, \varepsilon \varphi_{X}, \varepsilon^{2} \varphi_{X X}, \ldots\right) \varepsilon^{k} q_{k X}(X)
$$

а эволюция $J^{\nu}(X)$ в точках многообразия $\mathscr{M}^{\prime}$ с координатами $\mathbf{J}(X), \theta_{0}^{*}(X)$ запишется соответственно в виде

$$
\begin{aligned}
J_{\tau^{\mu}}^{\nu}(X)=\varepsilon & \left(\left\langle A_{1}^{\nu \mu}\right\rangle(\mathbf{J}(X)) q_{X}(X)+\frac{\partial\left\langle Q^{\nu \mu}\right\rangle(\mathbf{J}(X))}{\partial X} q(X)\right) \\
& +\varepsilon^{2} f^{\nu \mu}\left[\mathbf{J}, \theta_{0}^{*}, q(X), \varepsilon\right], \quad \nu, \mu=1, \ldots, N
\end{aligned}
$$

где $f^{\nu \mu}$ - регулярный при $\varepsilon \rightarrow 0$ асимптотический ряд по $\varepsilon^{k}$ с коэффициентами локальными функционалами от $q(X), \mathbf{J}(X)$ и $\theta_{0}^{*}(X)$.

ЛЕмма 3. В силу системы $(66)(\nu=1, \ldots, N, \mu$ фиксировано), описьвающей әволюиию $\mathbf{J}(X)$ согласно $(61)$ в точках $\mathscr{M}^{\prime}$, в рассматриваемой нами ситуачии выполняются соотночения

$$
k_{\tau^{\mu}}^{\alpha}(\mathbf{J}(X))=\varepsilon\left(q(X) \omega_{\mu}^{\alpha}(\mathbf{J}(X))\right)_{X}+\varepsilon^{2} \tilde{k}_{\mu}^{\alpha}\left[\mathbf{J}, \theta_{0}^{*}, q(X), \varepsilon\right],
$$

где $\boldsymbol{\omega}_{\nu}=\left(\omega_{\nu}^{1}, \ldots, \omega_{\nu}^{m}\right)$ - введенные в $(12)$ функции, $\tilde{k}_{\mu}^{\alpha}-$ регулярные при $\varepsilon \rightarrow 0$ функииональ $\mathbf{J}, \theta_{0}^{*}$ и $q(X)$ (линейные по $q(X)$ ).

ДокАЗАтЕльство. Система (61) допускает построение семейства решений, аналогичного описанному в методе Уизема, т.е. семейства решений вида

$$
\boldsymbol{\varphi}=\boldsymbol{\varphi}\left(\frac{\boldsymbol{\sigma}(X, T)}{\varepsilon}+\theta, X, T, \varepsilon\right)=\sum_{k \geqslant 0} \boldsymbol{\varphi}_{k}\left(\frac{\boldsymbol{\sigma}(X, T)}{\varepsilon}+\theta, X, T\right) \varepsilon^{k},
$$

$T=\varepsilon \tau^{\mu}$. В разложениях типа (68) мы не будем исследовать вопрос о сходимости получаемых степенных рядов, поскольку везде в дальнейшем достаточно будет, чтобы сумма первых нескольких членов получаемого ряда удовлетворяла локально в некоторой окрестности рассматриваемых $X$ и $T$ с точностью до величин более высокого порядка по $\varepsilon$ соотношениям, накладываемым на формальный ряд, что всегда будет иметь место по построению ряда. Ряд (68) мы здесь также рассматриваем как формальный, определяемый локально в точках, где $q(X) \neq 0$. 
Подставляя (68) в (61), в нулевом приближении получим, что $\varphi_{0}(\theta, X, T)$ при любом $T$ принадлежит $\mathscr{M}^{\prime}$, так как удовлетворяет одной из систем (12) (мы предполагаем здесь, что $I^{\mu}$ не является оператором импульса или аннулятором скобки (3), для которых утверждение леммы очевидно), причем

$$
\sigma_{T}^{\alpha}=q(X) \omega_{\mu}^{\alpha}(\mathbf{U}(X)), \quad \sigma_{X}^{\alpha}=k^{\alpha}(\mathbf{U}(X)) .
$$

Мы предполагаем, как уже было сказано ранее, что для систем (11), порожденных функционалами $I^{\mu}$ (кроме аннуляторов и оператора импульса), и соответствующих им подмногообразий $\mathscr{M}_{\boldsymbol{\omega}_{\mu}, \mathbf{k}}$ выполняются условия невырожденности А) и В), позволяюшие построить асимптотический ряд (68). При этом условия разрешимости системы на $\varphi_{1}(\theta, X, T)$, т.е. $N-m$ уравнений, выражающих ортогональность невязки $f_{1}$ левым собственным векторам соответствующего оператора $\hat{\mathbf{L}}_{\left[\mathbf{U}(X), \theta_{0}(X)\right]}$, вместе с уравнениями

$$
\mathbf{k}(\mathbf{U})_{T}=\left(q(X) \boldsymbol{\omega}_{\mu}(\mathbf{U})\right)_{X},
$$

следуюшими из (69), дадут, как и раньше, систему уравнений на $\mathbf{U}(X), \theta_{0}(X)$.

Нетрудно видеть (учитьвая (69)), что система на $\boldsymbol{\Phi}_{(1)}(\theta, X, T)$ здесь имеет вид

$$
\begin{aligned}
q(X)( & \left.\hat{\mathbf{L}}_{\left[\mathbf{U}(X), \theta_{0}(X)\right]}^{\mu} \boldsymbol{\Phi}_{1}\right)(\theta, X, T)=q(X)\left[\sum_{\mathbf{n}} \alpha_{j(\mathbf{n})}^{i(\mu)}\left(\boldsymbol{\sigma}_{X}, \boldsymbol{\Phi}_{(0)}, \boldsymbol{\Phi}_{(0) \theta^{\alpha}}, \ldots\right)\right. \\
& \left.\times\left(\boldsymbol{\Phi}_{(0) \mathbf{n} \theta, U^{\nu}} U_{X}^{\nu}+\boldsymbol{\Phi}_{(0) \mathbf{n} \theta, \theta^{\alpha}} \theta_{0 X}^{\alpha}\right)+\beta_{\alpha}^{i(\mu)}\left(\boldsymbol{\sigma}_{X}, \boldsymbol{\Phi}_{(0)}, \boldsymbol{\Phi}_{(0) \theta^{\alpha}}, \ldots\right) \boldsymbol{\sigma}_{X X}\right] \\
& +q_{X}(X) \widetilde{Q}_{\mu}^{i}\left(\boldsymbol{\Phi}_{(0)}, \boldsymbol{\sigma}_{X} \mathbf{\Phi}_{(0) \theta^{\alpha}}, \ldots\right)-\left(\boldsymbol{\Phi}_{(0) U^{\nu}}^{i} U_{T}^{\nu}+\boldsymbol{\Phi}_{(0) \theta^{\alpha}}^{i} \theta_{0 T}^{\alpha}\right)
\end{aligned}
$$

здесь все обозначения совпадают с обозначениями в $(26)$, величины $\sigma_{T}$ и $\sigma_{X}$ связаны с параметрами $\mathbf{U}(X)$, соответсвуюшими $\boldsymbol{\Phi}_{(0)}(\theta, X, T)$, соотношениями (69), функции $\alpha_{j(\mathbf{n})}^{i(\mu)}$ и $\beta_{\alpha}^{j(\mu)}$ те же самые, что и в методе усреднения Уизема для эволюционной системы, порожденной оператором $I^{\nu}$.

Последнее свойство позволяет заключить, что здесь, как и в методе Уизема, условия ортогональности невязки $f_{1}$ левым собственным векторам $\hat{\mathbf{L}}$ не накладывают никаких ограничений на параметры $\theta_{0}(X, T)$ и имеют, таким образом, вид

$$
q(X) A_{\nu}^{\zeta}(\mathbf{U}) U_{X}^{\nu}+B_{\nu}^{\zeta}(\mathbf{U}) U_{T}^{\nu}+q_{X}(X) C^{\zeta}(\mathbf{U})=0, \quad \zeta=1, \ldots, N-m .
$$

Вместе с условиями (70) система (71) дает $N$ уравнений на функции $\mathbf{U}(X)$.

При заданных гладких начальных данных система $(70),(71)$ имеет гладкое решение при $T<T_{0}$ (до момента опрокидывания волн), и в этой области, как и в методе Уизема, по функции $\varphi_{(0)}(\theta, X, T)$ из $\mathscr{M}^{\prime}$, характеризующейся величинами $\mathbf{U}(X, T)$ и $\theta_{0}(X, T)$, однозначно строятся последуюшие члены $\varphi_{(k)}(\theta, X, T)$ ряда $(68)$, являюшиеся, таким образом, функционалами $\mathbf{U}(X, T)$ и $\theta_{0}(X, T)$. Учитывая этот факт и воспользовавшись определением функционалов $\mathbf{J}(X)$ (55) и параметров $\mathbf{U}(X)(10)$, на семействе (68) решений системы (61) нетрудно получить соотношения

$$
J^{\nu}(X, T)=U^{\nu}(X, T)+\sum_{k \geqslant 1} \varepsilon^{k} \tilde{J}_{(k)}^{\nu}\left[\mathbf{U}, \theta_{0}\right],
$$


где $\mathbf{U}(X, T)$ и $\theta_{0}(X, T)$ - параметры, относяшиеся к функции $\varphi_{(0)}(\theta, X, T)$, принадлежашей $\mathscr{M}^{\prime}$, а $\mathbf{J}(X)$ - значения соответствуюших функционалов на решениях (68), отвечающих данным $\mathbf{U}(X, T)$ и $\theta_{0}(X, T)$.

После подстановки $\varphi(\theta, X, T)$ в виде (68) в выражение (65) и последующего интегрирования по $\theta$ получим выражение для эволюции интегралов $\mathbf{J}(X)$ на решениях (68), которое имеет вид

$$
J_{\tau^{\mu}}^{\nu}(X)=\varepsilon\left(q_{X}(X)\left\langle A^{\nu \mu}\right\rangle(\mathbf{U}(X))+q(X) \partial_{X}\left\langle Q^{\nu \mu}\right\rangle(\mathbf{U}(X))\right)+\varepsilon^{2} \tilde{f}^{\nu \mu}\left[\mathbf{U}, \theta_{0}, \varepsilon\right] .
$$

Отсюда, сравнивая (73) и (72), заключаем, что система

$$
U_{T}^{\nu}=q_{X}(X)\left\langle A_{1}^{\nu \mu}\right\rangle(\mathbf{U}(X))+q(X) \partial_{X}\left\langle Q^{\nu \mu}\right\rangle(\mathbf{U}(X)) \quad\left(T=\varepsilon \tau^{\mu}\right)
$$

эквивалентна (70), (71) и, таким образом, соотношения (67) являются следствием (66). Лемма доказана.

СлеДСТвИЕ. На функииях семейства $\mathscr{M}^{\prime}$ с координатами $\mathbf{J}(X), \theta_{0}^{*}(X)$ при $\varepsilon \rightarrow 0$ выполняются условия

$$
\begin{aligned}
\left\{k^{\alpha}(X), J^{\mu}(Y)\right\}=\varepsilon & \left(\omega_{\mu}^{\alpha}(\mathbf{J}(X)) \delta^{\prime}(X-Y)+\frac{\partial \omega_{\mu}^{\alpha}(\mathbf{J}(X))}{\partial X} \delta(X-Y)\right) \\
& +\varepsilon^{2} k^{\alpha \mu}\left[\mathbf{J}, \theta_{0}^{*}, \varepsilon\right],
\end{aligned}
$$

где $k^{\alpha \mu}-$ регулярнье при $\varepsilon \rightarrow 0$ функциональ $\mathbf{J}(X)$ и $\theta_{0}^{*}(X)$.

ЛЕмма 4. При выполнении сформулированного ранее предположения о том, что количество параметров $U^{\nu}$ равно $2 m+p$, где $p$ - количество аннуляторов скобки (3), имеют место соотношения

$$
\sum_{\nu=1}^{N} \frac{\partial k^{\alpha}(\mathbf{U})}{U^{\nu}} \omega_{\nu}^{\beta}(\mathbf{U}) \equiv 0 \quad \forall \alpha, \beta .
$$

ДокАЗАТЕЛЬСТво. Как уже указывалось ранее, любые из $m+1$ потоков, порождаемых $m+1$ интегралами из набора (8), линейно зависимы на семействе $m$-фазных решений системы (2), поскольку для некоторых $\lambda_{s}^{\nu}(\mathbf{U}), \nu=1, \ldots, N$, $s=1, \ldots, N-m$, на $\mathscr{M}^{\prime}$ вьполняются $N-m$ независимых соотношений

$$
\sum_{\nu=1}^{N} \lambda_{s}^{\nu}(\mathbf{U}) \omega_{\nu}^{\alpha}(\mathbf{U})=0, \quad \alpha=1, \ldots, m,
$$

где $\omega_{\nu}^{\alpha}(\mathbf{U})$ - введенные в (12) частоты, соответствующие интегралам $I^{\nu}$. Отсюда следует, что на $m$-фазных решениях $(2)$, соответствуюших параметрам $\mathbf{U}(X)$, для некоторых функций $\mu_{s}^{q}(\mathbf{U})$ выполняются соотношения

$$
\sum_{\nu=1}^{N} \lambda_{s}^{\nu}(\mathbf{U}) \delta I[\varphi]=\sum_{q=1}^{p} \mu_{s}^{q}\left(\mathbf{U}, \theta_{0}\right) \delta N_{q},
$$

где $N_{q}$ - аннуляторы скобки (3). Обозначим значения аннуляторов $N_{q}$ на $m$-фазных решениях (2) через $n_{q}(\mathbf{U})$ (зависимость от $\theta_{0}$ отсутствует в силу коммутирования $N_{q}$ с функционалами $I^{\nu}$, порождающими линейную зависимость начальных 
фаз от времени). При этом значения функционалов $I^{\nu}$ на $m$-фазных решениях (2) есть параметры $U^{\nu}$, и бесконечно малому сдвигу по $\mathscr{M}$ вдоль направления $\vec{\xi}$ (в пространстве параметров на $\mathscr{M})$, касательному к подмногообразию $\mathbf{k}(\mathbf{U})=$ const, $\mathbf{k}=\left(k^{1}, \ldots, k^{m}\right)$, соответствуют равномерно ограниченные вариации функций $\varphi^{i}(x)$. Тогда в силу $(77)$ получаем на $\mathscr{M}$

$$
\sum_{\nu=1}^{N} \lambda_{s}^{\nu}(\mathbf{U}) d U^{\nu}-\sum_{q=1}^{p} \mu_{s}^{q}\left(\mathbf{U}, \theta_{0}\right) d n_{q}(\mathbf{U})=0
$$

при $d k^{\alpha}=0$.

Отсюда следует, что $\mu\left(\mathbf{U}, \theta_{0}\right)=\mu(\mathbf{U})$ (зависимость от $\theta_{0}$ отсутствует), и для некоторых функций $\tau_{s}^{\alpha}(\mathbf{U}), \alpha=1, \ldots, m, s=1, \ldots, N-m$, выполняются соотношения

$$
\sum_{\nu=1}^{N} \lambda_{s}^{\nu}(\mathbf{U}) d U^{\nu}=\sum_{q=1}^{p} \mu_{s}^{q}(\mathbf{U}) d n_{q}(\mathbf{U})+\sum_{\alpha=1}^{m} \tau_{s}^{\alpha}(\mathbf{U}) d k^{\alpha}(\mathbf{U}) .
$$

Выражая $N-m=m+p$ дифференциалов $d n_{q}(\mathbf{U})$ и $d k^{\alpha}(\mathbf{U})$ из $N-m$ уравнений (78) (мы предполагаем, что в случае общего положения это можно сделать), получим соотношения

$$
\begin{aligned}
& d k^{\alpha}(\mathbf{U})=\sum_{s=1}^{N-m} a^{\alpha s}(\mathbf{U}) \sum_{\nu=1}^{N} \lambda_{s}^{\nu}(\mathbf{U}) d U^{\nu}, \\
& d n_{q}(\mathbf{U})=\sum_{s=1}^{N-m} b_{q}^{s}(\mathbf{U}) \sum_{\nu=1}^{N} \lambda_{s}^{\nu}(\mathbf{U}) d U^{\nu}
\end{aligned}
$$

для некоторых функций $a^{\alpha s}(\mathbf{U})$ и $b_{q}^{s}(\mathbf{U})$, после чего из $(76)$ непосредственно следует утверждение леммы. Лемма доказана.

Нетрудно видеть, что при сформулированных в лемме 4 условиях для функций $n_{q}(\mathbf{U})$ в силу (76) и (80) выполняются условия, аналогичные (75), т.е. имеет место

ЛЕмма 5. При сформулированных в лемме 4 условиях имеем соотношение $N=2 m+p$, где $p$ - число аннуляторов скобки (3) на подмногообразии $\mathscr{M}$, соответствующем полному семейству т-фазных решений (2), и выполняются условия

$$
\sum_{\nu=1}^{N} \frac{\partial n_{q}(\mathbf{U})}{\partial U^{\nu}} \omega_{\nu}^{\beta} \equiv 0
$$

СЛЕДСТВИЕ. На подмногообразии $\mathscr{M}^{\prime}$ в координатах $\left(\mathbf{J}(X), \theta_{0}^{*}(X)\right)$ имеют место соотношения

$$
\begin{aligned}
& \left\{k^{\alpha}(\mathbf{J}(X)), k^{\beta}(\mathbf{J}(Y))\right\}=\varepsilon^{2} \rho^{\alpha \beta}\left[\mathbf{J}, \theta_{0}^{*}, \varepsilon\right] \\
& \left\{k^{\alpha}(\mathbf{J}(X)), n_{q}(\mathbf{J}(Y))\right\}=\varepsilon^{2} v_{q}^{\alpha}\left[\mathbf{J}, \theta_{0}^{*}, \varepsilon\right]
\end{aligned}
$$

где $\rho^{\alpha \beta}, v_{q}^{\alpha}-$ регулярные при $\varepsilon \rightarrow 0$ функциональ $\mathbf{J}(X) u \theta_{0}^{*}(X)$. 
Действительно, воспользовавшись (74) и (75), получим

$$
\begin{aligned}
& \left\{k^{\alpha}(\mathbf{J}(X)), k^{\beta}(\mathbf{J}(Y))\right\}=\varepsilon\left(\sum_{\nu=1}^{N} \omega_{\nu}^{\alpha}(\mathbf{J}(X)) \frac{\partial k^{\beta}(\mathbf{J})}{\partial J^{\nu}}(X) \delta^{\prime}(X-Y)\right. \\
& \left.\quad+\left(\omega_{\nu}^{\alpha}(\mathbf{J}(X)) \frac{\partial k^{\beta}(\mathbf{J})}{\partial J^{\nu}}(X)\right)_{X} \delta(X-Y)\right)+\varepsilon^{2} k_{\nu}^{\alpha}\left[\mathbf{J}, \theta_{0}^{*}, \varepsilon\right] \frac{\partial k^{\beta}(\mathbf{J})}{\partial J^{\nu}}(Y) \\
& =\varepsilon^{2} k_{\nu}^{\alpha}\left[\mathbf{J}, \theta_{0}^{*}, \varepsilon\right] \frac{\partial k^{\beta}(\mathbf{J})}{\partial J^{\nu}}(Y) .
\end{aligned}
$$

Равенство (83) доказывается аналогично.

Займемся вычислением скобок $\left\{J^{\nu}(X), \theta_{0}^{* \alpha}(Y)\right\}$ и $\left\{\theta_{0}^{* \alpha}(X), \theta_{0}^{\beta *}(Y)\right\}$ на $\mathscr{M}^{\prime}$. Как уже было сказано вьше, функционалы типа $\int q(X) J^{\nu}(X) d X$ порождают в силу скобки (45) локальные потоки вида (61). При этом эволюция плотностей $A^{\nu}\left(\varphi, \varphi_{\theta^{\alpha}}, \ldots\right)$ интегралов $a^{\nu}(X)$, вводимых в (49) для определения координат $U^{\nu}(X)$ в окрестности $\mathscr{M}^{\prime}$, также имеет вид, аналогичный $(61)$, т.е.

$$
\begin{aligned}
A_{\tau^{\mu}}^{\nu}(\theta, X)= & q(X) S_{\mu}^{\nu}\left(\boldsymbol{\varphi}, \varphi_{\theta^{\alpha}}, \ldots, \varepsilon \boldsymbol{\varphi}_{X}, \varepsilon \boldsymbol{\varphi}_{\theta^{\alpha} X}, \ldots\right) \\
& +\varepsilon q_{X} \widetilde{S}_{\mu}^{\nu}\left(\boldsymbol{\varphi}, \theta_{\theta^{\alpha}}, \ldots, \varepsilon \boldsymbol{\varphi}_{X}, \varepsilon \boldsymbol{\varphi}_{\theta^{\alpha} X}, \ldots\right)+\ldots
\end{aligned}
$$

После подстановки в это выражение функций $\varphi(\theta, X)$ в виде

$$
\boldsymbol{\varphi}(\theta, X)=\boldsymbol{\Phi}_{\mathrm{in}}\left(\theta+\theta_{0}^{*}(X)+\frac{1}{\varepsilon} \int_{X_{0}}^{X} \mathbf{k}\left(\mathbf{J}\left(X^{\prime}\right)\right) d X^{\prime}, \mathbf{U}\left[\mathbf{J}, \theta_{0}^{*}, \varepsilon\right]\right)
$$

и интегрирования по $\theta$ получим выражение для эволюции $a^{\nu}(X)$ на функциях из $\mathscr{M}^{\prime}$ с координатами $\mathbf{J}(X)$ и $\theta_{0}^{*}(X)$ в виде

$$
a_{\tau^{\mu}}^{\nu}(X)=\sum_{k \geqslant 0} \bar{r}_{\mu(k)}^{\nu}\left[\mathbf{J}, \theta_{0}^{*}, q(X)\right](X) \varepsilon^{k} .
$$

Очевидно, что аналогичные соотношения будут выполняться и для эволюции функционалов $\mathbf{U}(X)$ на $\mathscr{M}^{\prime}$, выражаюшихся через $\mathbf{a}(X)$ точечными заменами, причем поскольку система (61) на функциях из $\mathscr{M}^{\prime}$ вида (84) порождает в нулевом порядке по $\varepsilon$ сдвиг начальных фаз $\theta_{0}^{\alpha}$ по времени, не затрагивая остальных параметров, то нулевой член в этом разложении отсутствует и, таким образом, скобки Пуaсcона $U^{\nu}(X)$ и $J^{\mu}(Y)$ на $\mathscr{M}^{\prime}$ будут как функции координат $\mathbf{J}(X)$ и $\theta_{0}^{*}(X)$ иметь вид

$$
\left.\left\{U^{\nu}(X), J^{\mu}(Y)\right\}\right|_{\mathbf{G}(\theta, X)=0}=\sum_{k \geqslant 1} r_{\mu(k)}^{\nu}\left[\mathbf{J}, \theta_{0}^{*}\right] \varepsilon^{k},
$$

т.е. $\left.\left\{U^{\nu}(X), J^{\mu}(Y)\right\}\right|_{\mathscr{M}^{\prime}}=O(\varepsilon)$ при $\varepsilon \rightarrow 0$ в координатах $\left(\mathbf{J}(X), \theta_{0}^{*}(X)\right)$.

ЛЕмма 6. При $Y \neq X_{0}$ на функииях из $\mathscr{M}^{\prime}$ с координатами $\mathbf{J}(X), \theta_{0}^{*}(X)$ имеют место соотношения

$$
\left\{\theta_{0}^{\alpha *}(X), J^{\nu}(Y)\right\}=\varepsilon \sigma^{\alpha \beta}\left[\mathbf{J}, \theta_{0}^{*}, \varepsilon\right],
$$

где $\sigma^{\alpha \beta}-$ регулярные при $\varepsilon \rightarrow 0$ функииональ $\mathbf{J} и \theta_{0}^{*}$. 
ДоКАЗАТЕЛЬСТво. Введем функционалы $\vartheta^{* \alpha}(X)$ по формуле

$$
\begin{aligned}
\vartheta^{* \alpha}(X)= & \frac{1}{(2 \pi)^{m}} \int_{0}^{2 \pi} \cdots \int_{0}^{2 \pi} \sum_{i} \varphi^{i}(\theta, X) \\
& \times \Phi_{\operatorname{in} \theta^{\alpha}}^{i}\left(\theta+\frac{1}{\varepsilon} \int_{X_{0}}^{X} \mathbf{k}\left(\mathbf{J}\left(X^{\prime}\right)\right) d X^{\prime}, \mathbf{U}[\varphi](X)\right) d^{m} \theta .
\end{aligned}
$$

Нетрудно видеть, что функционалы $\theta_{0}^{* \alpha}(X)$ в силу своего определения (см. (56)) выражаются теми же (не зависящими от $\varepsilon$ ) функциями $\tau^{\alpha}\left(\boldsymbol{\vartheta}^{*}(X)\right)$ через $\vartheta^{* \alpha}(X)$, которыми функционалы $\theta_{0}^{\alpha}(X)$ выражаются через функции $\vartheta_{0}(X)$, введенные в (50). Формулы

$$
\theta_{0}^{* \alpha}(X)=\tau^{\alpha}\left(\boldsymbol{\vartheta}^{*}(X)\right)
$$

при этом относятся к карте на $\mathscr{M}^{\prime}$, в которой $\theta_{0}^{*}(X)$ принимает при любом $\varepsilon$ те же (т.е. не зависяшие от $\varepsilon$ ) значения, что и $\theta_{0}(X)$ в карте, к которой относятся функции $\tau^{\alpha}$ в определении $\theta_{0}^{\alpha}(X)$ через $\vartheta_{0}(X)$.

Эволюция функционалов (87) в силу потоков (61), порождаемых функционалами вида $\int q(X) J^{\mu}(X) d X$, задается эволюцией полей $\varphi(\theta, X)$ согласно системе (61), а также эволюцией функционалов $\mathbf{J}(X)$ и $\mathbf{U}[\varphi](X)$, присутствующих в $\boldsymbol{\Phi}_{\text {in }}$, задаваемой в координатах $\mathbf{J}(X)$ и $\theta_{0}^{*}(X)$ на $\mathscr{M}^{\prime}$ формулами $(66)$ и $(85)$. Отсюда после подстановки $\varphi(\theta, X)$ в виде $(84)$ в систему (61) и интегрирования по $\theta$, устраняющего нерегулярность при $\varepsilon \rightarrow 0$ в сдвиге аргумента $\theta$, можно, как и в случае с $\mathbf{U}(X)$, заключить, что скобки Пуассона $\theta^{* \alpha}(X)$ с $J^{\mu}(Y)$ на $\mathscr{M}^{\prime}$ являются (в силу (88)) регулярными при $\varepsilon \rightarrow 0$ функциями $\mathbf{J}(X)$ и $\theta_{0}^{*}(X)$, т.е.

$$
\left\{\theta_{0}^{* \alpha}(X), J^{\mu}(Y)\right\}=\sum_{k \geqslant 0} \varepsilon^{k} \lambda^{\alpha \mu}\left[\mathbf{J}, \theta_{0}^{*}\right] .
$$

Пусть теперь $Y \neq X_{0}$. Рассмотрим функционалы вида $\int q\left(Y^{\prime}\right) J^{\mu}\left(Y^{\prime}\right) d Y^{\prime}$ с функциями $q\left(Y^{\prime}\right)$, имеюшими носитель вблизи $Y$, так что $q\left(X_{0}\right)=0$. Нетрудно видеть, принимая во внимание утверждение (67) леммы 3, что при этом эволюция функционалов (87) в силу соответствующих потоков на функциях из $\mathscr{M}^{\prime}$ с координатами $\mathbf{J}$ и $\theta_{0}^{*}$ в нулевом порядке по $\varepsilon$ при $\varepsilon \rightarrow 0$ будет иметь вид

$$
\begin{aligned}
\vartheta_{\tau^{\mu}}^{* \alpha}(X)= & \frac{1}{(2 \pi)^{m}} \int_{0}^{2 \pi} \cdots \int_{0}^{2 \pi}\left[q(X) Q_{\mu}\left(\boldsymbol{\Phi}_{\mathrm{in}}, k^{\beta}(\mathbf{J}) \boldsymbol{\Phi}_{\mathrm{in} \theta^{\beta}}, \ldots\right) \boldsymbol{\Phi}_{\mathrm{in} \theta^{\alpha}}\right. \\
& \left.+\boldsymbol{\Phi}_{\mathrm{in}} \int_{X_{0}}^{X}\left(\omega_{\mu}^{\beta}\left(\mathbf{J}\left(X^{\prime}\right)\right) q\left(X^{\prime}\right)\right)_{X^{\prime}} d X^{\prime} \times \boldsymbol{\Phi}_{\mathrm{in} \boldsymbol{\theta}^{\alpha} \boldsymbol{\theta}^{\beta}}\right] d^{m} \theta \\
= & \frac{1}{(2 \pi)^{m}} \int_{0}^{2 \pi} \cdots \int_{0}^{2 \pi} q(X) \omega_{\mu}^{\beta}(\mathbf{J}(X))\left[\boldsymbol{\Phi}_{\mathrm{in} \theta^{\beta}} \boldsymbol{\Phi}_{\mathrm{in} \theta^{\alpha}}+\boldsymbol{\Phi}_{\mathrm{in}} \boldsymbol{\Phi}_{\mathrm{in} \boldsymbol{\theta}^{\alpha} \boldsymbol{\theta}^{\beta}}\right] d^{m} \theta=0
\end{aligned}
$$

(после подстановки $\varphi(\theta, X)$ в виде (84) мы воспользовались свойством (12) для функций $\left.Q_{\mu}^{i}\right)$. Отсюда непосредственно следует утверждение леммы.

Для $\mathbf{J}\left(X_{0}\right)$ при этом (в точке $X_{0}$ отсутствует зависящий от $\varepsilon$ сдвиг начальной фазы $\theta_{0}$ ) аналогичным образом нетрудно получить на $\mathscr{M}^{\prime}$ (в координатах $\left.\mathbf{J}(X), \theta_{0}^{*}(X)\right)$

$$
\left\{\theta^{* \alpha}(X), J^{\mu}\left(X_{0}\right)\right\}=\omega_{\mu}^{\alpha}\left(\mathbf{J}\left(X_{0}\right)\right) \delta\left(X-X_{0}\right)+O(\varepsilon)
$$


(в силу того, что в нулевом порядке по $\varepsilon$ функционалы $\int q(X) J^{\mu}(X) d X$ порождают линейную зависимость начальных фаз $\theta_{0}(X)$ от времени с частотами $q(X) \boldsymbol{\omega}_{\mu}(X)$, а в точке $X_{0}$ имеем $\left.\theta_{0}^{*}\left(X_{0}\right) \equiv \theta_{0}\left(X_{0}\right)\right)$. При этом для функционалов $k^{\alpha}(\mathbf{J}(X))$ в силу (75) при любом $Y$, включая $X_{0}$, в координатах $\mathbf{J}(X), \theta_{0}^{*}(X)$ на $\mathscr{M}^{\prime}$ будут иметь место соотношения

$$
\left.\left\{\theta_{0}^{*}(X), k^{\alpha}(\mathbf{J}(Y))\right\}\right|_{\mathscr{M}^{\prime}}=O(\varepsilon), \quad \varepsilon \rightarrow 0 .
$$

Пользуясь (82) и (90), нетрудно получить, что скобка Пуассона функционалов $\vartheta^{* \alpha}(X)$ между собой регулярна на $\mathscr{M}^{\prime}$ в координатах $\mathbf{J}(X), \theta_{0}^{*}(X)$ при $\varepsilon \rightarrow 0$, и, следовательно, то же свойство выполняется и для скобок вида $\left\{\theta_{0}^{* \alpha}(X), \theta_{0}^{\beta *}(Y)\right\}$, т.е.

$$
\left.\left\{\theta_{0}^{* \alpha}(X), \theta_{0}^{\beta *}(Y)\right\}\right|_{\mathscr{M}^{\prime}}=\gamma^{\alpha \beta}\left[\mathbf{J}, \theta_{0}^{*}, \varepsilon\right](X, Y),
$$

где $\gamma^{\alpha \beta}(X, Y)$ - регулярньй при $\varepsilon \rightarrow 0$ функционал $\mathbf{J}$ и $\theta_{0}^{*}$. Более подробная информация о скобках этого вида нам не понадобится.

Перейдем теперь к процедуре ограничения по Дираку скобки (45) на подмногообразие $\mathscr{M}^{\prime}$, пользуясь координатами $\mathbf{J}(X), \theta_{0}^{*}(X)$ и $G^{i}(\theta, X)$ в окрестности $\Delta_{\delta}$.

Для ограничения по Дираку скобки Пуассона (45) на $\mathscr{M}^{\prime}$ нам необходимо подобрать к функционалам $J^{\nu}(X)$ и $\theta_{0}^{* \alpha}(X)$ добавки вида

$$
\begin{aligned}
V^{\nu}(X) & =\frac{1}{(2 \pi)^{m}} \int_{0}^{2 \pi} \cdots \int_{0}^{2 \pi} \int v_{i}^{\nu}(X, Y, \theta, \varepsilon) G^{i}(\theta, Y) d^{m} \theta d Y, \\
W^{\alpha}(X) & =\frac{1}{(2 \pi)^{m}} \int_{0}^{2 \pi} \cdots \int_{0}^{2 \pi} \int w_{i}^{\alpha}(X, Y, \theta, \varepsilon) G^{i}(\theta, Y) d^{m} \theta d Y
\end{aligned}
$$

такие, чтобы потоки, порожденные функционалами $\tilde{J}^{\nu}(X)=J^{\nu}(X)+V^{\nu}(X)$ и $\tilde{\theta}_{0}^{\alpha}(X)=\theta_{0}^{* \alpha}(X)+W^{\alpha}(X)$, оставляли $\mathscr{M}^{\prime}$ инвариантным, и затем положить на $\mathscr{M}^{\prime}$

$$
\begin{aligned}
\left\{J^{\nu}(X), J^{\mu}(Y)\right\}^{*} & =\left.\left\{\tilde{J}^{\nu}(X), \tilde{J}^{\mu}(Y)\right\}\right|_{\mathscr{M}^{\prime}}\left(\mathbf{J}, \theta_{0}^{*}\right), \\
\left\{\theta_{0}^{* \alpha}(X), \theta^{\beta *}(Y)\right\}^{*} & =\left.\left\{\tilde{\theta}_{0}^{\alpha}(X), \tilde{\theta}_{0}^{\beta}(Y)\right\}\right|_{\mathscr{M}^{\prime}}\left(\mathbf{J}, \theta_{0}^{*}\right), \\
\left\{J^{\nu}(X), \theta_{0}^{* \alpha}(Y)\right\}^{*} & =\left.\left\{\tilde{J}^{\nu}(X), \tilde{\theta}_{0}^{* \alpha}(Y)\right\}\right|_{\mathscr{M}^{\prime}}\left(\mathbf{J}, \theta_{0}^{*}\right) .
\end{aligned}
$$

Для функций $v_{i}^{\nu}(X, Y, \theta, \varepsilon)$ и $w_{i}^{\alpha}(X, Y, \theta, \varepsilon)$ получаем соотношения

$$
\begin{aligned}
\frac{1}{(2 \pi)^{m}} & \int_{0}^{2 \pi} \cdots \int_{0}^{2 \pi} \int v_{j}^{\nu}\left(Y, Z, \theta^{\prime}, \varepsilon\right) \\
& \times\left.\left\{G^{i}(\mathbf{U}[\varphi](X), \varphi(\theta, X), \ldots), G^{j}\left(\mathbf{U}[\varphi](Z), \varphi\left(\theta^{\prime}, Z\right), \ldots\right)\right\} d^{m} \theta^{\prime} d Z\right|_{\mathscr{M}^{\prime}} \\
=- & \left.\left\{G^{i}(\mathbf{U}[\varphi](X), \varphi(\theta, X), \ldots), J^{\nu}[\varphi](Y)\right\}\right|_{\mathscr{M}^{\prime}} \\
& \quad \frac{1}{(2 \pi)^{m}} \int_{0}^{2 \pi} \cdots \int_{0}^{2 \pi} \int w_{j}^{\alpha}\left(Y, Z, \theta^{\prime}, \varepsilon\right) \\
& \times\left.\left\{G^{i}(\mathbf{U}[\varphi](X), \varphi(\theta, X), \ldots), G^{j}\left(\mathbf{U}[\varphi](Z), \varphi\left(\theta^{\prime}, Z\right), \ldots\right)\right\} d^{m} \theta^{\prime} d Z\right|_{\mathscr{M}^{\prime}} \\
=- & \left.\left\{G^{i}(\mathbf{U}[\varphi](X), \varphi(\theta, X), \ldots), \theta_{0}^{* \alpha}[\varphi](Y)\right\}\right|_{\mathscr{M}^{\prime}}
\end{aligned}
$$

При вычислении скобок Пуассона связей $G^{i}(\theta, X)$ с какими-либо величинами на $\mathscr{M}^{\prime}$ мы будем пользоваться тем обстоятельством, что непосредственно 
на $\mathscr{M}^{\prime}$ величины, стоящие в скобках определения связей $G^{i}(\theta, X)$ через $\varphi(X)$ (см. (53)), обрашаются в нуль, и, таким образом, при вычислении скобок типа $\left\{G^{i}(\theta, X), C\left(\theta^{\prime}, Z\right)\right\}$ на $\mathscr{M}^{\prime}$ можно не вычислять скобки ядер операторов $\hat{\mathbf{L}}$, присутствующих в $(53)$ с $C\left(\theta^{\prime}, Z\right)$, вынося ядра $\hat{\mathbf{L}}$ из-под знака скобок Пуассона согласно тождеству Лейбнища в виде множителей.

Учитывая соотношения (90), (82), а также (64), (86), (89) и (91) для функционалов $\mathbf{J}$ и $\theta_{0}^{*}$, входяших в (53), нетрудно видеть, что скобки Пуассона вида $\left\{G^{i}(\theta, X), G^{j}\left(\theta^{\prime}, Z\right)\right\}$, а также $\left\{G^{i}(\theta, X), J^{\nu}(Y)\right\}$ и $\left\{G^{i}(\theta, X), \theta_{0}^{* \alpha}(Y)\right\}$ на подмногообразии $\mathscr{M}^{\prime}$ с координатами $\mathbf{J}(X), \theta_{0}^{*}(X)$ в самой общей форме могут быть записаны регулярными при $\varepsilon \rightarrow 0$ асимптотическими рядами вида

$$
\begin{aligned}
& \left.\left\{G^{i}(\theta, X), G^{j}\left(\theta^{\prime}, Z\right)\right\}\right|_{\mathscr{M}^{\prime}}= \\
& =\frac{1}{(2 \pi)^{2 m}} \int_{0}^{2 \pi} \cdots \int_{0}^{2 \pi} \tilde{L}_{s\left[\mathbf{U}\left[\mathbf{J}, \theta_{0}^{*}, \varepsilon\right]\right]}^{i}\left(\theta+\theta_{0}^{*}(X)+\frac{\mathbf{s}(X)}{\varepsilon}, \tau+\theta_{0}^{*}(X)+\frac{\mathbf{s}(X)}{\varepsilon}, X\right) \\
& \quad \times\left(\sum_{\mathbf{n}, k \geqslant p \geqslant 0} M_{(k),(p), \mathbf{n}}^{s l}\left(\tau+\theta_{0}^{*}(X)+\frac{\mathbf{s}(X)}{\varepsilon},\left[\mathbf{J}, \theta_{0}^{*}\right]\right) \varepsilon^{k} \delta^{(p)}(X-Z) \delta_{\mathbf{n} \theta}(\boldsymbol{\tau}-\boldsymbol{\zeta})\right) \\
& \quad \times \tilde{L}_{l\left[\mathbf{U}\left[\mathbf{J}, \theta_{0}^{*}, \varepsilon\right]\right]}^{i}\left(\theta^{\prime}+\theta_{0}^{*}(Z)+\frac{\mathbf{s}(Z)}{\varepsilon}, \boldsymbol{\zeta}+\theta_{0}^{*}(Z)+\frac{\mathbf{s}(Z)}{\varepsilon}, Z\right) d^{m} \boldsymbol{\tau} d^{m} \boldsymbol{\zeta} .
\end{aligned}
$$

Здесь через $\mathbf{s}(X)$ и $\quad \mathbf{s}(Z)$ обозначены интегралы $\int_{X_{0}}^{X} \mathbf{k}\left(\mathbf{J}\left(X^{\prime}\right)\right) d X^{\prime}$ и $\int_{X_{0}}^{Z} \mathbf{k}\left(\mathbf{J}\left(X^{\prime}\right)\right) d X^{\prime}$, а также

$$
\begin{aligned}
& \left.\left\{G^{i}(\theta, X), \mathbf{J}^{\nu}(Y)\right\}\right|_{\mathscr{M}^{\prime}}= \\
& =\frac{1}{(2 \pi)^{m}} \int_{0}^{2 \pi} \cdots \int_{0}^{2 \pi} \tilde{L}_{s\left[\mathbf{U}\left[\mathbf{J}, \theta_{0}^{*}, \varepsilon\right]\right]}^{i}\left(\theta+\theta_{0}^{*}(X)+\frac{\mathbf{s}(X)}{\varepsilon}, \boldsymbol{\tau}+\theta_{0}^{*}(X)+\frac{\mathbf{s}(X)}{\varepsilon}, X\right) \\
& \quad \times\left(\sum_{k \geqslant p \geqslant 0} S_{(k),(p)}^{s \nu}\left(\tau+\theta_{0}^{*}(X)+\frac{\mathbf{s}(X)}{\varepsilon},\left[\mathbf{J}, \theta_{0}^{*}\right]\right) \varepsilon^{k} \delta^{(p)}(X-Y)\right) d^{m} \boldsymbol{\tau}, \\
& \left.\left\{G^{i}(\theta, X), \theta^{* \alpha}(Y)\right\}\right|_{\mathscr{M}^{\prime}}= \\
& =\frac{1}{(2 \pi)^{m}} \int_{0}^{2 \pi} \cdots \int_{0}^{2 \pi} \tilde{L}_{s\left[\mathbf{U}\left[\mathbf{J}, \theta_{0}^{*}, \varepsilon\right]\right]}^{i}\left(\theta+\theta_{0}^{*}(X)+\frac{\mathbf{s}(X)}{\varepsilon}, \boldsymbol{\tau}+\theta_{0}^{*}(X)+\frac{\mathbf{s}(X)}{\varepsilon}, X\right) \\
& \quad \times\left(\sum_{k \geqslant p \geqslant 0} T_{(k),(p)}^{s \alpha}\left(\tau+\theta_{0}^{*}(X)+\frac{\mathbf{s}(X)}{\varepsilon},\left[\mathbf{J}, \theta_{0}^{*}\right]\right) \varepsilon^{k} \delta^{(p)}(X-Y)\right) d^{m} \boldsymbol{\tau} .
\end{aligned}
$$

Здесь функции, зависяшие от $Z$ и $Y$, заменены на функции, зависяшие от $X$, согласно формулам типа $\varepsilon \delta^{\prime}(X-Z) f(Z)=\varepsilon f(X) \delta^{\prime}(X-Z)+\varepsilon f_{X}(X) \delta(X-Z)$. Легко видеть, что при этом каждое дифференцирование по $X$ входит с множителем $\varepsilon$.

Функции

$$
\begin{gathered}
\tilde{\boldsymbol{\kappa}}_{\left[\mathbf{U}\left[\mathbf{J}, \theta_{0}^{*}, \varepsilon\right]\right]}^{q}\left(\theta+\theta_{0}^{*}(X)+\frac{1}{\varepsilon} \int_{X_{0}}^{X} \mathbf{k}\left(\mathbf{J}\left(X^{\prime}\right)\right) d X^{\prime}, X\right), \\
\tilde{\boldsymbol{\kappa}}_{\left[\mathbf{U}\left[\mathbf{J}, \theta_{0}^{*}, \varepsilon\right]\right]}^{q}\left(\theta+\theta_{0}^{*}(Z)+\frac{1}{\varepsilon} \int_{X_{0}}^{Z} \mathbf{k}\left(\mathbf{J}\left(X^{\prime}\right)\right) d X^{\prime}, Z\right)
\end{gathered}
$$

являются на $\mathscr{M}^{\prime}$ соответственно левыми и правыми собственными векторами линейного оператора на пространстве $2 \pi$-периодических по $\theta$ функций с ядром 
$\left\{G^{i}(\theta, X), G^{j}\left(\theta^{\prime}, Z\right)\right\}$ с нулевыми собственными значениями. При этом функции $\boldsymbol{\kappa}_{\left[\mathbf{U}\left[\mathbf{J}, \theta_{0}^{*}, \varepsilon\right]\right]}\left(\theta+\theta_{0}^{*}(X)+(1 / \varepsilon) \int_{X_{0}}^{X} \mathbf{k}\left(\mathbf{J}\left(X^{\prime}\right)\right) d X^{\prime}, X\right)$, как нетрудно видеть из $(100)$ и (101), ортогональны также (при любом $\varepsilon$ ) и правым частям $(97),(98)$, и системы (97), (98), таким образом, являются в случае общего положения разрешимыми. Решения $v_{j}^{\nu}(X, Y, \theta)$ и $w_{j}^{\alpha}(X, Y, \theta)$ определяются с точностью до любой линейной комбинации векторов $\boldsymbol{\kappa}_{\left[\mathbf{U}\left[\mathbf{J}, \theta_{0}^{*}, \varepsilon\right]\right]}\left(\theta+\theta_{0}^{*}(Y)+(1 / \varepsilon) \int_{X_{0}}^{Y} \mathbf{k}\left(\mathbf{J}\left(X^{\prime}\right)\right) d X^{\prime}, Y\right)$, что, однако, не влияет в силу (54) ни на вид добавок $V^{\nu}(X)$ и $W^{\alpha}(X)$, ни на ограничение по Дираку скобки (45) на $\mathscr{M}^{\prime}$ согласно формулам (94)-(96), поскольку при взятии скобок $V^{\nu}(X)$ и $W^{\alpha}(X)$ с какими-либо величинами на $\mathscr{M}^{\prime}$ свертка этой линейной комбинации с соответствующим ядром $L_{s\left[\mathrm{U}\left[\mathbf{J}, \theta_{0}^{*}, \varepsilon\right]\right]}^{i}(\ldots)$ будет в силу уже сказанного выше вынесена из-под знака скобок Пуассона в виде множителя, обращающегося в нуль на $\mathscr{M}^{\prime}$.

Для однозначного определения функций

$$
\begin{aligned}
v_{j}^{\nu}(X, Y, \theta, \varepsilon) & =\bar{v}_{j}^{\nu}\left(X, Y, \theta+\theta_{0}^{*}(Y)+\frac{1}{\varepsilon} \int_{X_{0}}^{Y} \mathbf{k}\left(\mathbf{J}\left(X^{\prime}\right)\right) d X^{\prime}, \varepsilon\right), \\
w_{j}^{\alpha}(X, Y, \theta, \varepsilon) & =\bar{w}_{j}^{\alpha}\left(X, Y, \theta+\theta_{0}^{*}(Y)+\frac{1}{\varepsilon} \int_{X_{0}}^{Y} \mathbf{k}\left(\mathbf{J}\left(X^{\prime}\right)\right) d X^{\prime}, \varepsilon\right)
\end{aligned}
$$

наложим на них $N+m$ дополнительных условий, потребовав, чтобы они также были ортогональны при любых $X$ и $Y$ соответствуюшим функциям

$$
\tilde{\boldsymbol{\kappa}}_{\left[\mathbf{U}\left[\mathbf{J}, \theta_{0}^{*}, \varepsilon\right]\right]}^{q}\left(\theta+\theta_{0}^{*}(Y)+\frac{1}{\varepsilon} \int_{X_{0}}^{Y} \mathbf{k}\left(\mathbf{J}\left(X^{\prime}\right)\right) d X^{\prime}, Y, \varepsilon\right),
$$

т.е.

$$
\begin{aligned}
& \frac{1}{(2 \pi)^{m}} \int_{0}^{2 \pi} \cdots \int_{0}^{2 \pi} \sum_{j} \bar{v}_{j}^{\nu}(X, Y, \theta, \varepsilon) \tilde{\boldsymbol{\kappa}}_{j\left[\mathbf{U}\left[\mathbf{J}, \theta_{0}^{*}, \varepsilon\right]\right]}(\theta, Y, \varepsilon) d^{m} \theta=0 \\
& \frac{1}{(2 \pi)^{m}} \int_{0}^{2 \pi} \cdots \int_{0}^{2 \pi} \sum_{j} \bar{w}_{j}^{\alpha}(X, Y, \theta, \varepsilon) \tilde{\boldsymbol{\kappa}}_{j\left[\mathbf{U}\left[\mathbf{J}, \theta_{0}^{*}, \varepsilon\right]\right]}^{q}(\theta, Y, \varepsilon) d^{m} \theta=0
\end{aligned}
$$

для любых $X, Y, \varepsilon$ на $\mathscr{M}^{\prime}$.

После подстановки величин (104) и (105) в систему (97), (98) для функций $\bar{v}_{j}^{\nu}$ и $\bar{w}_{j}^{\alpha}$ получим линейные неоднородные системы, представимые в виде регулярных при $\varepsilon \rightarrow 0$ асимптотических рядов. Величины $\left\{G^{i}(\theta, X), G^{j}\left(\theta^{\prime}, Z\right)\right\}$, $\left\{G^{i}(\theta, X), J^{\nu}(Y)\right\}$ и $\left\{G^{i}(\theta, X), \theta_{0}^{* \alpha}(Y)\right\}$ подставляются при этом в виде $(99),(100)$ и $(101)$ соответственно и после взятия в $(97),(98)$ всех производных по $X$, входящих каждый раз с множителем $\varepsilon$, нерегулярный при $\varepsilon \rightarrow 0$ сдвиг фаз

$$
\theta_{0}^{*}(X)+\frac{1}{\varepsilon} \int_{X_{0}}^{X} \mathbf{k}\left(\mathbf{J}\left(X^{\prime}\right)\right) d X^{\prime}
$$

во всех функциях, зависящих от $\theta$, может быть опущен. Как уже отмечалось выше, в случае общего положения, соответствующем невырожденности матрицы скобок Пуассона связей в конечномерном случае, эти системы являются разрешимыми при любом $\varepsilon$, и при наличии дополнительных условий (107), (108) функции 
$\bar{v}(X, Y, \theta, \varepsilon)$ и $\bar{w}(X, Y, \theta, \varepsilon)$ могут быть однозначно определены на подмногообразии $\mathscr{M}^{\prime}$ с координатами $\mathbf{J}(X), \theta_{0}^{*}(X)$ (от которых системы $(97),(98)$ зависят как от параметров) в виде регулярных при $\varepsilon \rightarrow 0$ асимптотических рядов по степеням $\varepsilon$, T.e.

$$
\begin{aligned}
\bar{v}_{j}^{\nu}\left(X, Y, \theta,\left[\mathbf{J}, \theta_{0}^{*}\right], \varepsilon\right) & =\sum_{k \geqslant 0} \bar{v}_{j(k)}^{\nu}\left(X, Y, \theta,\left[\mathbf{J}, \theta_{0}^{*}\right]\right) \varepsilon^{k}, \\
\bar{w}_{j}^{\alpha}\left(X, Y, \theta,\left[\mathbf{J}, \theta_{0}^{*}\right], \varepsilon\right) & =\sum_{k \geqslant 0} \bar{w}_{j(k)}^{\alpha}\left(X, Y, \theta,\left[\mathbf{J}, \theta_{0}^{*}\right]\right) \varepsilon^{k} .
\end{aligned}
$$

ЗАмечаниЕ. Часто скобки Пуассона (3) для исходной системы (2) такие, как, например, скобка Гарднера-Захарова-Фаддеева

$$
\{\varphi(x), \varphi(y)\}=\delta^{\prime}(x-y)
$$

или скобка Магри-Ленарда

$$
\{\varphi(x), \varphi(y)\}_{M L}=-\frac{1}{2} \delta^{\prime \prime \prime}(x-y)+(\varphi(x)+\varphi(y)) \delta^{\prime}(x-y),
$$

имеют такой вид, что соответствующая им скобка (45) вырождена в нулевом порядке по $\varepsilon$ в координатах $\varphi(\theta, X)$. Это вырождение возникает из-за наличия в каждом из членов таких скобок производных $\delta$-функций по $x$ (т.е. операторов $\partial / \partial x$ ) или производных по $x$ функций $\varphi(x)$, входяших в $(45)$ с множителями $\varepsilon$. Однако во введенных выше координатах $J^{\nu}(X), \theta_{0}^{* \alpha}(X)$ и $G^{i}(\theta, X)$ в окрестности $\mathscr{M}^{\prime}$ операторы $\varepsilon \partial / \partial X$, применяемые к функциям вида $(75)$ на $\mathscr{M}^{\prime}$, содержат неисчезающий при $\varepsilon \rightarrow 0$ вклад вида $k^{\alpha}(\mathbf{J}(X)) \partial / \partial \theta^{\alpha}$, что позволяет в случае обшего положения (для связей общего вида) считать системы для функций $\bar{v}_{j}^{\nu}$ и $\bar{w}_{j}^{\alpha}$ невырожденными в нулевом порядке по $\varepsilon$, записывая для функций $\bar{v}_{j}^{\nu}$ и $\bar{w}_{j}^{\alpha}$ регулярные при $\varepsilon \rightarrow 0$ асимптотические разложения (109) и (110).

Более точно, разложения (109) и (110) имеют вид

$$
\begin{aligned}
\bar{v}_{j}^{\nu}(X, Y, \theta, \varepsilon) & =\sum_{k \geqslant p \geqslant 0} \bar{v}_{j(k),(p)}^{\nu}\left(X, \theta,\left[\mathbf{J}, \theta_{0}^{*}\right]\right) \varepsilon^{k} \delta^{(p)}(X-Y), \\
\bar{w}_{j}^{\alpha}(X, Y, \theta, \varepsilon) & =\sum_{k \geqslant p \geqslant 0} \bar{w}_{j(k),(p)}^{\alpha}\left(X, \theta,\left[\mathbf{J}, \theta_{0}^{*}\right]\right) \varepsilon^{k} \delta^{(p)}(X-Y) ;
\end{aligned}
$$

величины $V^{\nu}(X)$ и $W^{\alpha}(X)$, как нетрудно видеть, не содержат обобщенных функций. Подстановка (111) и (112) в соответствуюшие системы $(97),(98)$ даст в $k$-м порядке по $\varepsilon$ при любых заданных $X$ и $p$ (коэффициенты при $\delta$-функциях приравниваются так же, как и при степенях $\varepsilon$ ) линейные дифференциальные по $\theta$ неоднородные системы для функций $\bar{v}_{j(k),(p)}^{\nu}(X, \theta)$ и $\bar{w}_{j(k),(p)}^{\alpha}(X, \theta)$, зависящие от $\mathbf{J}$ и $\theta_{0}^{*}$ как от параметров. Правые части этих систем зависят при этом (линейным образом) от предыдущих $\overline{\mathbf{v}}_{\left(k^{\prime}\right),(q)}$ и $\overline{\mathbf{w}}_{\left(k^{\prime}\right),(q)} \mathrm{c} k^{\prime}<k$. При сделанных нами ранее предположенях описанные выше системы будут однозначно разрешимы и ряды (111), (112) могут быть построены методом последовательных приближений.

Как уже отмечалось вьше, потоки (61) на функциях вида (84) оставляют $\mathscr{M}^{\prime}$ инвариантным в нулевом порядке по $\varepsilon$, порождая на нем линейную зависимость начальных фаз от времен $\tau^{\mu}$, и, таким образом, в выражении (100) и соответственно в правой части системы $(97)$ отсутствует нулевой член по $\varepsilon\left(S_{0}^{s \nu} \equiv 0\right)$, что при 
выполнении предположения о невырожденности системы в нулевом порядке по $\varepsilon$ $\left(\right.$ в координатах $\mathbf{J}, \theta_{0}^{*}$ на $\left.\mathscr{M}^{\prime}\right)$ влечет $\bar{v}_{j(0),(p)}^{\nu} \equiv 0$, т.е. для функций $v_{j}^{\nu}$ и $w_{j}^{\alpha}$ окончательно имеем

$$
\begin{aligned}
& v_{j}^{\nu}\left(X, Y, \theta,\left[\mathbf{J}, \theta_{0}^{*}\right], \varepsilon\right)= \\
& \quad=\sum_{k \geqslant p \geqslant 1} \bar{v}_{j(k),(p)}^{\nu}\left(X, \theta+\theta_{0}^{*}(Y)+\frac{1}{\varepsilon} \int_{X_{0}}^{Y} \mathbf{k}\left(\mathbf{J}\left(X^{\prime}\right)\right) d X^{\prime},\left[\mathbf{J}, \theta_{0}^{*}\right]\right) \varepsilon^{k} \delta^{(p)}(X-Y), \\
& w_{j}^{\alpha}\left(X, Y, \theta,\left[\mathbf{J}, \theta_{0}^{*}\right], \varepsilon\right)= \\
& \quad=\sum_{k \geqslant p \geqslant 0} \bar{w}_{j(k),(p)}^{\alpha}\left(X, \theta+\theta_{0}^{*}(Y)+\frac{1}{\varepsilon} \int_{X_{0}}^{Y} \mathbf{k}\left(\mathbf{J}\left(x^{\prime}\right)\right) d X^{\prime},\left[\mathbf{J}, \theta_{0}^{*}\right]\right) \varepsilon^{k} \delta^{(p)}(X-Y) .
\end{aligned}
$$

Более подробная информация о функциях $v_{j}^{\nu}$ и $w_{j}^{\alpha}$ нам не понадобится.

Сформулируем и докажем теперь основной результат.

ТЕОРЕМа 1. Пусть для системы (2), гамильтоновой относительно скоб-

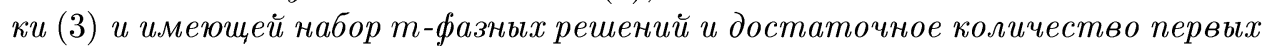
интегралов типа (8), выполняются все ранее перечисленные свойства общего положсения относительно функииональной независимости параметров $U^{\nu}$ на этом семействе и возможсности выражения через них параметров $\mathbf{k}, \boldsymbol{\omega}$ u $\mathbf{r}$, а также соотношения, связывающие количество аннуляторов скобки (3) с числом введенных ранее дополнительных параметров $r^{1}, \ldots, r^{g}$.

Тогда при выполнении условий регулярности А) и В) введенных ранее подмногообразий $\mathscr{M}_{\boldsymbol{\omega} \mathbf{k}}^{\nu}$ и аналогичных условий для подмногообразия $\mathscr{M}$, а также условия невырожсденности скобок Пуассона связей $\left\{G^{i}(\theta, X), G^{j}\left(\theta^{\prime}, Z\right)\right\}$ на $\mathscr{M}^{\prime}$ в нулевом порядке по є в координатах $\mathbf{J}, \theta_{0}^{*}$, позволяющего построить функиии $v_{j}^{\nu}$ и $w_{j}^{\alpha}$ в виде асимптотических рядов (113), (114), имеем:

1) скобка Дубровина-Новикова, определяемая формулой (38):

$$
\left\{U^{\nu}(X), U^{\mu}(Y)\right\}=\left\langle A_{1}^{\nu \mu}\right\rangle(\mathbf{U}(X)) \delta^{\prime}(X-Y)+\frac{\partial\left\langle Q^{\nu \mu}\right\rangle}{\partial U^{\lambda}}(\mathbf{U}(X)) U_{X}^{\lambda} \delta(X-Y),
$$

удовлетворяет тождеству Якоби;

2) для скобки (38) выполняются соотношения

$$
\begin{gathered}
\left\{k^{\alpha}(\mathbf{U}(X)), k^{\beta}(\mathbf{U}(Y))\right\}=0, \quad\left\{k^{\alpha}(\mathbf{U}(X)), n_{q}(\mathbf{U}(Y))\right\}=0, \\
\left\{k^{\alpha}(\mathbf{U}(X)), U^{\mu}(Y)\right\}=\omega_{\nu}^{\alpha}(\mathbf{U}(X)) \delta^{\prime}(X-Y)+\omega_{\nu X}^{\alpha}(\mathbf{U}(X)) \delta(X-Y) .
\end{gathered}
$$

ДоКАЗАТЕЛЬСТво. При выполнении вышеперечисленных условий можно с помощью описанной процедуры произвести ограничение скобки Пуассона (45) на подмногообразие $\mathscr{M}^{\prime}$ с координатами $\mathbf{J}(X), \theta_{0}^{*}(X)$. При этом согласно формуле $(42)$ будем иметь для ограниченной на $\mathscr{M}^{\prime}$ скобки следуюшие формулы:

$$
\begin{aligned}
& \left\{J^{\nu}(X), J^{\mu}(Y)\right\}^{*}=\left.\left\{J^{\nu}(X), J^{\mu}(Y)\right\}\right|_{\mathscr{M}^{\prime}}\left(\mathbf{J}, \theta_{0}^{*}\right) \\
& \quad-\frac{1}{(2 \pi)^{2 m}} \int_{0}^{2 \pi} \cdots \int_{0}^{2 \pi} \iint v_{i}^{\nu}(X, \bar{X}, \theta, \varepsilon) \times\left\{G^{i}(\theta, \bar{X}), G^{j}(\theta, \bar{Y})\right\} \\
& \quad \times v_{j}^{\mu}\left(Y, \bar{Y}, \theta^{\prime}, \varepsilon\right) d \bar{X} d \bar{Y} d^{m} \theta d^{m} \theta^{\prime},
\end{aligned}
$$


и аналогичные формулы для скобок $\left\{J^{\nu}(X), \theta_{0}^{* \alpha}(Y)\right\}^{*}$ и $\left\{\theta_{0}^{* \alpha}(X), \theta_{0}^{* \beta}(Y)\right\}^{*}$ (с заменой функций $v_{i}^{\nu}, v_{j}^{\mu}$ на $w_{i}^{\alpha}, w_{j}^{\beta}$ для функций $\theta_{0}^{* \alpha}(X)$ и $\left.\theta_{0}^{* \beta}(Y)\right)$. Нетрудно видеть, что здесь, как и раньше, при интегрировании функций (113), (114), а также (99), нерегулярньй при $\varepsilon \rightarrow 0$ сдвиг начальных фаз $\theta_{0}^{*}(X)+(1 / \varepsilon) \int_{X_{0}}^{X} \mathbf{k}\left(\mathbf{J}\left(X^{\prime}\right)\right) d X^{\prime}$, присутствующий во всех функциях, зависящих от $\theta$ и $\theta^{\prime}$ (после взятия интегралов по $d \bar{X}$ и $d \bar{Y})$, оказывается несушественньг, и скобка Пуассона $\{\ldots, \ldots\}^{*}$ на $\mathscr{M}^{\prime}$, таким образом, регулярна при $\varepsilon \rightarrow 0$ в координатах $\mathbf{J}(X)$ и $\theta_{0}^{*}(X)$. При этом, используя соотношения (64), (86), (91), а также соотношения (113) и (114), получим для скобки $\{\ldots, \ldots\}^{*}$ в координатах $\mathbf{J}, \theta_{0}^{*}$ соотношения

$$
\begin{aligned}
& \left\{J^{\nu}(X), J^{\mu}(Y)\right\}^{*}=\varepsilon\left(\left\langle A_{1}^{\nu \mu}\right\rangle(\mathbf{J}(X)) \delta^{\prime}(X-Y)\right. \\
& \left.+\left(\frac{\partial}{\partial X}\left\langle Q^{\nu \mu}\right\rangle(\mathbf{J}(X))\right) \delta(X-Y)\right)+O\left(\varepsilon^{2}\right)
\end{aligned}
$$

и

$$
\left\{J^{\nu}(X), \theta_{0}^{* \alpha}(Y)\right\}^{*}=O(\varepsilon)
$$

при $X \neq X_{0}$,

$$
\left\{\theta_{0}^{* \alpha}(X), \theta_{0}^{* \beta}(Y)\right\}^{*}=O(1)
$$

при $\varepsilon \rightarrow 0$.

Отсюда видно, что тождества Якоби, содержащие только функционалы $\mathbf{J}$ в точках $X, Y, Z$, не совпадаюших с $X_{0}$, совпадают в первом неисчезающем порядке по $\varepsilon$ (при $\varepsilon^{2}$ ) с соответвующими тождествами Якоби для скобки (38) на пространстве полей $U^{\nu}(X)$. Учитывая теперь то, что точка $X_{0}$ была выбрана произвольно, а выражение (38) от $X_{0}$ не зависит, получим, что скобка (38) удовлетворяет тождеству Якоби. Ее кососимметричность при этом тривиально следует из кососимметричности скобки (3). Соотношения (115), (116) следуют при этом из $(82),(83)$ и (74) соответственно. Теорема доказана.

ТЕОРема 2. Пусть при сформулированных в теореме 1 предположениях имеются два несовпадающих набора $\left\{I^{1}, \ldots, I^{N}\right\}$ u $\left\{\bar{I}^{1}, \ldots, \bar{I}^{N}\right\}$ из $N$ первых интегралов системы (2) вида (8) (некоторые из интегралов этих наборов могут совпадать).

Тогда скобки Дубровина-Новикова, полученные с помощью этих наборов, совпадают, т.е. если $U^{\nu}=\left\langle\mathscr{P}^{\nu}\right\rangle, \bar{U}^{\nu}=\left\langle\overline{\mathscr{P}}^{\nu}\right\rangle$, где $\mathscr{P}^{\nu}, \overline{\mathscr{P}}^{\nu}-$ плотности интегралов первого и второго наборов соответственно, то преобразование скобки (38), полученной с помощью набора $\left\{I^{1}, \ldots, I^{N}\right\}, \kappa$ координатам $\bar{U}^{\nu}(X)$ на подмногообразии $\mathscr{M}:$

$$
\bar{U}^{\nu}=\bar{u}^{\nu}(\mathbf{U})
$$

даст скобку Дубровина-Новикова, полученную с помощью набора $\left\{\bar{I}^{1}, \ldots, \bar{I}^{N}\right\}$.

ДоКАЗАТЕЛЬСТВо. В рассматриваемой нами ситуации общего положения ограничение по Дираку скобки (45) на подмногообразие $\mathscr{M}^{\prime}$ определено однозначно, т.е. скобки (117)-(119), полученные с помошью координат $\left(\mathbf{J}, \theta_{0}^{*}\right)$ и $\left(\overline{\mathbf{J}}, \theta_{0}^{*}\right)($ соответствующих первому и второму наборам интегралов) в окрестности $\mathscr{M}^{\prime}$, переходят друг в друга при соответствующих преобразованиях друг в друга координат 
$\left(\mathbf{J}, \theta_{0}^{*}\right)$ и $\left(\overline{\mathbf{J}}, \theta_{0}^{*}\right)$ на $\mathscr{M}^{\prime}$. При этом в силу (59) и аналогичного соотношения для $\overline{\mathbf{U}}$ и $\left(\overline{\mathbf{J}}, \theta_{0}^{*}\right)$ можно заключить, что переход от координат $\mathbf{J}(X)$ к $\overline{\mathbf{J}}(X)$ имеет вид

$$
\bar{J}^{\nu}(X)=\bar{u}^{\nu}(\mathbf{J}(X))+\sum_{k \geqslant 1} \varepsilon^{k} \bar{J}_{(k)}^{\nu}\left(\mathbf{J}, \mathbf{J}_{X}, \ldots, \mathbf{J}_{k X}, \theta_{0 X}^{*}, \ldots, \theta_{0 k X}^{*}\right),
$$

и, таким образом, переход от скобок $(117)$ к скобкам $\left\{\bar{J}^{\nu}(X), \bar{J}^{\mu}(Y)\right\}$ при такой замене координат совпадает (при $X, Y \neq X_{0}$ ) в первом неисчезающем по $\varepsilon$ порядке (при первой степени $\varepsilon$ ) с соответствующим преобразованием скобки Дубровина-Новикова в силу точечной замены (120), что и доказывает теорему.

Эволюция плотностей $\mathscr{P}^{\nu}(x)$ в силу потоков $(11)$, порожденных интегралами $I^{\mu}$, имеет (см. (36)) вид

$$
\mathscr{P}_{\tau^{\mu}}^{\nu}\left(\varphi, \varphi_{x}, \ldots\right)=\partial_{x} Q^{\nu \mu}\left(\varphi, \varphi_{x}, \ldots\right)
$$

в то время как потоки, порождаемые функционалами $\int U^{\mu}(X) d X$ на пространстве полей $\mathbf{U}(X)$ в силу скобки Дубровина-Новикова, записьваются в виде

$$
U_{T^{\mu}}^{\nu}=\partial_{X}\left\langle Q^{\nu \mu}\right\rangle(\mathbf{U})
$$

и являются, таким образом (см. (30)), уравнениями Уизема для $m$-фазных решений гамильтоновой системы, порожденной функционалом $I^{\mu}$, не являющимся оператором импульса или аннулятором скобки (3) (напомним, что все такие системы имеют обшее семейство $m$-фазных решений).

Все такие потоки коммутируют между собой в силу коммутации функционалов $\int J^{\mu}(X) d X$ относительно скобки Дубровина-Новикова, и, кроме того, то же самое верно и для потоков, порожденных интегралами по $X$ от усредненных плотностей любых функционалов, имеюших вид (8) и коммутируюших с гамильтонианом и интегралами $I^{\nu}$, поскольку любой такой функционал может быть включен в набор $\left\{I^{\nu}\right\}$ вместо любого из присутствующих там интегралов $I^{\nu}$, что согласно теореме 2 не изменит скобки (38). Интегралы по $X$ от усредненной плотности функционала импульса (5) и аннуляторов скобки (3), имеющих вид (8), порождают в силу скобки (38) сдвиг по $X$ и нулевые потоки соответственно. Интеграл по $X$ от усредненной плотности гамильтониана (4) порождает уравнения Уизема для $m$-фазных решений системы (2).

В заключение автор благодарит С. П. Новикова за постановку задачи, а также И. М. Кричевера, О.И. Мохова, М. В. Павлова и Е. В. Ферапонтова за плодотворные обсуждения.

\section{Список литературы}

1. Уизем Джс. Линейные и нелинейные волны. М.: Мир, 1977.

2. Luke J. C. A perturbation method for nonlinear dispersive wave problems // Proc. Roy. Soc. London. Ser. A. 1966. V. 292. № 1430. P. 403-412.

3. Дубровин Б. А., Новиков С. П. Гамильтонов формализм одномерных систем гидродинамического типа и метод усреднения Боголюбова-Уизема // ДАН СССР. 1983. Т. 270. № 4. C. 781-785.

4. Дубровин Б. А., Новиков С. П. Гидродинамика слабо деформированных солитонных решеток. Дифференциальная геометрия и гамильтонова теория // УМН. 1989. Т. 44. № 6. C. $29-98$.

5. Доброхотов С. Ю., Маслов В.П. Конечнозонные почти периодические решения в ВКБ-приближениях // Итоги науки и техники. Современные проблемы математики. Т. 15. М.: ВИНИТИ, 1980. 
6. Кричевер И. М. Метод усреднения для двумерных "интегрируемых" уравнений. // Функц. анализ и его прилож. 1988. Т. 22. № 3. С. 37-52.

7. Кричевер И. М. Спектральная теория двумерных периодических операторов и ее приложения // УМН. 1989. Т. 44. № 2. С. 121-184.

8. Krichever I. M. Perturbation Theory in Periodic Problems for Two-Dimensional Integrable Systems // Sov. Sci. Rev. Section C. 1992. V. 9.

9. Царев С. П. О скобках Пуассона и одномерных гамильтоновых системах гидродинамического типа // ДАН СССР. 1985. Т. 282. №3. С. 534-537.

10. Ferapontov E. $V$. On integrability of $3 \times 3$ semi-Hamiltonian hydrodynamic type systems $u_{t}^{i}=v_{j}^{i}(u) u_{x}^{j}$ which do not possess Riemann invariants // Physica D. 1993. V. 63. P. 50-70.

11. Ferapontov E. V. On the matrix Hopf equation and integrable Hamiltonian systems of hydrodynamic type, which do not possess Riemann invariants // Physics Letters A. 1993. V. 179. P. 391-397.

12. Новиков С.П., Мальцев А.Я. Лиувиллева форма усредненных скобок Пуассона // УМН. 1993. Т. 48. № 1. С. 155, 156.

13. Мохов О. И. Локальные скобки Пуассона третьего порядка // УМН. 1985. Т. 40. № 5. C. 257,258 .

14. Мохов О.И. Гамильтоновы дифференциальные операторы и контактная геометрия // Функц. анализ и его прилож. 1987. Т. 21. № 3. С. 53-60.

15. Гринберг Н.И. О скобках Пуассона гидродинамического типа с вырожденной метрикой // УМН. 1985. Т. 40. № 4. С. 217, 218.

16. Мальцев A.Я., Павлов М.В. О методе усреднения Уизема // Функц. анализ и его прилож. 1995. Т. 29. № 1. С. 7-24.

Институт теоретической физики

Поступило в редакцию им. Л. Д. Ландау РАН,

ул. Косыгина 2, Москва 117940.

27.VIII.1997

E-mail: maltsev@itp.ac.ru 